https://helda.helsinki.fi

\title{
Transancestral GWAS of alcohol dependence reveals common genetic underpinnings with psychiatric disorders
}

\section{3andMe Res Team}

2018-12

23andMe Res Team \& Räikkönen, K 2018 , ' Transancestral GWAS of alcohol dependence reveals common genetic underpinnings with psychiatric disorders ' , Nature Neuroscience , vol. 21 , no. 12 , pp. 1656-+ . https://doi.org/10.1038/s41593-018-0275-1

http://hdl.handle.net/10138/307699

https://doi.org/10.1038/s41593-018-0275-1

publishedVersion

Downloaded from Helda, University of Helsinki institutional repository.

This is an electronic reprint of the original article.

This reprint may differ from the original in pagination and typographic detail.

Please cite the original version. 


\title{
Transancestral GWAS of alcohol dependence reveals common genetic underpinnings with psychiatric disorders
}

\begin{abstract}
Liability to alcohol dependence (AD) is heritable, but little is known about its complex polygenic architecture or its genetic relationship with other disorders. To discover loci associated with AD and characterize the relationship between AD and other psychiatric and behavioral outcomes, we carried out the largest genome-wide association study to date of DSM-IV-diagnosed AD. Genome-wide data on 14,904 individuals with AD and 37,944 controls from 28 case-control and family-based studies were meta-analyzed, stratified by genetic ancestry (European, $n=46,568$; African, $n=6,280$ ). Independent, genome-wide significant effects of different $A D H 1 B$ variants were identified in European (rs1229984; $P=9.8 \times 10^{-13}$ ) and African ancestries (rs2066702; $P=2.2 \times 10^{-9}$ ). Significant genetic correlations were observed with 17 phenotypes, including schizophrenia, attention deficit-hyperactivity disorder, depression, and use of cigarettes and cannabis. The genetic underpinnings of AD only partially overlap with those for alcohol consumption, underscoring the genetic distinction between pathological and nonpathological drinking behaviors.
\end{abstract}

xcessive alcohol use is a leading contributor to morbidity and mortality. One in 20 deaths worldwide is attributable to alcohol consumption, as is $5.1 \%$ of the global burden of disease ${ }^{1}$. $\mathrm{AD}$, as defined by the Fourth Edition of the American Psychiatric Association's Diagnostic and Statistical Manual of Mental Disorders $(\mathrm{DSM}-\mathrm{IV})^{2}$, is a serious psychiatric disorder characterized by tolerance, withdrawal, loss of control over drinking, and excessive alcohol consumption despite negative health and social consequences. Among alcohol drinkers, $12 \%$ meet criteria for DSM-IV AD during their lifetimes ${ }^{3}$. In the United States, only $25 \%$ of those with AD ever receive treatment ${ }^{4}$.

AD is moderately heritable ( $49 \%$ by a recent meta-analysis) ${ }^{5}$ and numerous genome-wide association studies (GWAS) have aimed to identify loci contributing to this genetic variance (see ref. ${ }^{6}$ for a review). According to one study, common single-nucleotide polymorphisms (SNPs) are responsible for as much as $30 \%$ of the variance in $\mathrm{AD}^{7}$, but few have been identified to date. Variants in the genes responsible for alcohol metabolism, especially $A D H 1 B$ and $A L D H 2$, have been strongly implicated ${ }^{8-13}$. The association between $\mathrm{AD}$ (and related drinking phenotypes) and rs1229984, a missense SNP (Arg48His) in ADH1B that affects the conversion of alcohol to acetaldehyde, represents one of the largest common-variant effect sizes observed in psychiatry, with the His 48 allele accelerating ethanol metabolism and affording an approximately threefold reduction in likelihood of $\mathrm{AD}$ across numerous studies ${ }^{8,10}$. Another functional polymorphism, rs671 in ALDH2 (Glu504Lys), strongly affects alcohol metabolism by blocking conversion of acetaldehyde to acetate and has an even stronger effect on risk for $\mathrm{AD}$, but is rare except in some Asian populations ${ }^{8,12,13} A D H 1 B$ and $A L D H 2$ polymorphisms, however, only explain a small proportion of the heritable variation in $\mathrm{AD}$ in populations of European or African ancestry.

In this study, the Substance Use Disorders working group of the Psychiatric Genomics Consortium (PGC-SUD ${ }^{14}$ ) compiled the largest numbers of carefully diagnosed alcohol-dependent individuals and alcohol-exposed controls to date, from both casecontrol and family studies. These included substantial numbers of people with European ancestry (EU, $n=46,568$, including 38,686 unrelated individuals) and people with admixed African-American ancestry (AA, $n=6,280$, including 5,799 unrelated individuals) subjects. $\mathrm{AD}$ diagnoses were derived from clinician ratings or semistructured interviews following DSM-IV ${ }^{2}$ criteria. Each study was subjected to stringent quality control before conducting GWAS within each population of each study, followed by a genome-wide meta-analysis. We estimated the SNP-heritability $\left(h_{g}^{2}\right)$ of $\mathrm{AD}$ and examined the extent to which aggregate genetic variation in $\mathrm{AD}$ is related to traits from 45 other GWAS, including continuous measures of alcohol consumption. We also examined whether polygenic risk scores (PRS) derived from these analyses predicted alcohol dependence and related measures of problem drinking in three independent samples.

\section{Results}

GWAS meta-analyses. The transancestral discovery meta-analysis of GWAS of AD in 28 cohorts (Table 1 and Supplementary Table 1) identified a genome-wide significant (GWS; $P<5 \times 10^{-8}$ ) association in the $A D H$ gene cluster on chromosome 4 (Fig. 1 and Table 2). Examining this locus in each population (Fig. 2), rs1229984 in $A D H 1 B$ was the strongest associated variant from the analysis in $\mathrm{EU}\left(z=-7.13, P=9.8 \times 10^{-13}\right)$, while rs2066702, also in $A D H 1 B$, was the most significant variant in AA $\left(z=-5.98, P=2.2 \times 10^{-9}\right)$. Transancestral modeling reinforced the robust effects of rs1229984 and other $A D H 1 B$ SNPs on liability to $\mathrm{AD}$ across inverse-variance weighted, random effects, and Bayesian models (Supplementary Fig. 1 and Supplementary Table 2).

Clumping the $A D H$ locus for linkage disequilibrium (LD; $r^{2}<0.1$ within $500 \mathrm{~kb}$ ) suggested multiple independent signals in both populations, with the differing leading alleles reflecting different LD structures and allele frequencies in each population (Table 2 and Supplementary Fig. 2). Conditional analyses controlling for rs1229984 and rs2066702 had limited power, but results showed limited attenuation of effect sizes between marginal and conditional analyses, consistent with the existence of additional independent effects in the region (Supplementary Table 3 and Supplementary Fig. 3). Suggestive independent signals in the 


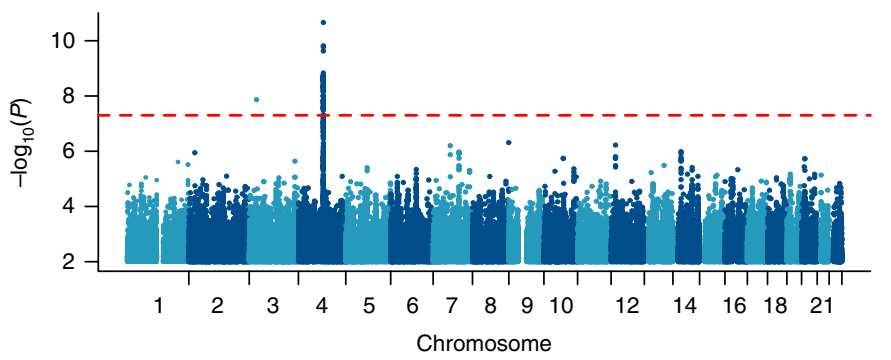

Fig. 1 | Manhattan plot of discovery transancestral meta-analysis showing strong evidence for rs1229984 in ADH1B. Results from the discovery metaanalysis of all cohorts ( $\left.n_{\text {case }}=14,904 ; n_{\text {control }}=37,944\right)$ for association of genome-wide SNPs with AD under a fixed effects meta-analysis weighted by effective sample size. Dashed red reference line indicates GWS after correction for multiple testing $\left(P<5 \times 10^{-8}\right)$.

genotyped cohorts included trialleleic variant rs894368 (marginal $z=-4.57, P=4.9 \times 10^{-6}$; conditional $\left.z=4.53, P=5.8 \times 10^{-6}\right)$ and insertion rs112346244 (marginal odds ratio $=0.912$, s.e. $=0.024$, $z=-3.81, P=1.4 \times 10^{-4}$; conditional odds ratio $=0.883$, s.e. $=0.025$, $z=-5.05, P=4.5 \times 10^{-7}$; Supplementary Table 3). Several additional variants that were prioritized in the conditional analysis, while not significant, were in moderate to strong LD with rs698 (marginal odds ratio $=1.115$, s.e. $=0.021, z=5.19, P=2.1 \times 10^{-7}$; conditional odds ratio $=1.084$, s.e. $\left.=0.021, z=3.78, P=1.6 \times 10^{-4}\right)$, a functional $A D H 1 C$ variant with a role in $\mathrm{AD}^{8,11}$.

A single novel SNP on chromosome 3, rs7644567, also reached GWS in the meta-analysis $\left(z=5.68, P=1.36 \times 10^{-8}\right.$; Supplementary Fig. 4). Potential biological associations with rs7644567, including chromatin contacts (Supplementary Fig. 5) and cerebellar expression of $R B M S 3$, are summarized in Supplementary Methods. However, rs7644567 did not replicate in two independent AA samples (YalePenn 2 and Collaborative Study on the Genetics of Alcoholism (COGA) African-American Family GWAS (AAfGWAS)) or the independent FINRISK cohort; all three replication cohorts estimated effects of the minor allele in the opposite direction of the discovery meta-analysis (Supplementary Table 4; see Supplementary Information). The SNP is also rare in most EU samples (minor allele frequency (MAF) <0.01), with the current GWAS results primarily attributable to AA cohorts, along with the Finnish Twin cohort (FinnTwin) and the Finnish Nicotine Addiction Genetics Project (NAG-Fin). The EU cohorts in the discovery meta-analysis show no evidence of association of $\mathrm{AD}$ with the SNPs in strongest $\mathrm{LD}$ with rs7644567 in African (rs13098461; $z=0.27, P=0.79$ ) or Finnish (rs9854300; $z=0.10, P=0.92$ ) reference samples (Supplementary Methods). Based on the clear lack of replication, there is insufficient evidence to conclude that rs7644567 is associated with $\mathrm{AD}$ based on the current results.

There was limited genome-wide evidence for heterogeneity across all cohorts, within ancestry, between ancestries, or between study designs within ancestry (Supplementary Methods and Supplementary Figs. 6-8). Evidence for inflation from population stratification or other confounding was also limited in the discovery meta-analysis $(\lambda=0.962$; Supplementary Fig. 9) and within EU $(\lambda=1.053$, LD score regression (LDSR) intercept $=1.018)$ and AA $(\lambda=1.007$, LDSR intercept $=0.991-0.997$; Supplementary Methods $)$. Gene-level association testing with MAGMA ${ }^{15}$ did not identify any additional significant genes in EU or AA (Supplementary Table 5 and Supplementary Methods), likely due to lack of power.

Heritability and genetic correlations. Liability-scale SNPheritability of AD was estimated at $h_{g}^{2}=0.090$ (s.e. $=0.019, z=4.80$, $\left.P=8.02 \times 10^{-7}\right)$ in the meta-analysis of unrelated EU samples.
Exclusion of the $A D H 1 B$ locus did not substantially modify this estimate $\left(h_{g}^{2}=0.089\right.$, s.e. $\left.=0.0185\right)$. Nominally significant polygenic signal for the meta-analysis of unrelated AA individuals was observed based on LDSR with scores computed from 1000 Genomes Project African populations $(z=2.12, P=0.017)$, but the quantitative estimate of $h^{2}$ was unstable depending on the choice of reference panel, reflecting the challenge of correctly specifying LDSR and robustly modeling LD for the AA population (Supplementary Methods).

Significant genetic correlation with $\mathrm{AD}$ in $\mathrm{EU}$ was observed for 17 traits after correction for multiple testing $\left(P<1.11 \times 10^{-3}\right.$ for 45 tested traits; Fig. 3 and Supplementary Table 6$)$. The largest positive correlations were with smoking initiation $\left(r_{g}=0.708\right.$, s.e. $=0.134$, $\left.P=1.3 \times 10^{-7}\right)$ and lifetime cannabis initiation $\left(r_{g}=0.793\right.$, s.e. $=0.217$, $\left.P=2.5 \times 10^{-4}\right)$, as well as with other psychiatric disorders, especially schizophrenia $\left(r_{g}=0.357\right.$, s.e. $\left.=0.054, P=3.2 \times 10^{-11}\right)$, attention deficit-hyperactivity disorder $\left(r_{g}=0.444\right.$, s.e. $\left.=0.097, P=4.2 \times 10^{-6}\right)$, and depression $\left(r_{g}=0.561\right.$, s.e. $\left.=0.085, P=3.5 \times 10^{-11}\right)$. Educational attainment $\left(r_{g}=-0.468\right.$, s.e. $\left.=0.066, P=9.7 \times 10^{-13}\right)$ and age at first birth (higher values indicate that participants were older when they had their first child; $r_{g}=-0.626$, s.e. $=0.104, P=2.0 \times 10^{-9}$ ) showed significant inverse genetic correlation with $\mathrm{AD}$, suggesting that liability to $\mathrm{AD}$ risk was genetically related to lower educational attainment and lower age at which the participant had his or her first child.

Unexpected patterns of genetic correlation were observed when comparisons were made to other alcohol-related measures, indicating that those measures reflect aspects of alcohol use that are genetically distinguishable. AD was genetically correlated with alcohol consumption in a meta-analysis of the Alcohol Genomewide Association (AlcGen) and Cohorts for Aging and Research in Genomic Epidemiology Plus (CHARGE +$)$ consortia $^{16}\left(r_{g}=0.695\right.$, s.e. $\left.=0.155, P=6.9 \times 10^{-6}\right)$, but only modestly with alcohol consumption from the recent large UK Biobank analysis ${ }^{17}\left(r_{g}=0.371\right.$, s.e. $\left.=0.092, P=5.2 \times 10^{-5}\right)$. No significant genetic correlation was observed between $\mathrm{AD}$ and a recent GWAS of the alcohol use disorders identification test (AUDIT) in a 23andMe $\operatorname{cohort}^{18}\left(r_{g}=0.076\right.$, s.e. $=0.171, P=0.656$ ), perhaps due to the low levels of drinking and drinking-related problems in that population ${ }^{18}$. $\mathrm{AD}$ is, however, nominally genetically correlated with GWAS of delay discounting in the 23andMe sample ${ }^{19}\left(r_{g}=0.487\right.$, s.e. $\left.=0.178, P=6.0 \times 10^{-3}\right)$.

Association with $A D H 1 B$ expression. Based on the strong observed association with rs1229984 and rs2066702, we examined whether other variants affecting $A D H 1 B$ expression (expression quantitative trait loci) were also associated with AD using GTEx v7 results (https://www.gtexportal.org/)20. Three variants, rs11939328 (EU, $P=0.78$; AA $P=0.98$; transancestral (trans), $P=0.78$ ), rs 10516440 (EU, $P=3.97 \times 10^{-6}$; AA, $P=1.97 \times 10^{-3}$; trans, $P=4.72 \times 10^{-8}$ ), and rs7664780 (EU, $P=0.87$; AA, $P=0.083$; trans, $P=0.405$ ), were selected after LD-informed clumping and the exclusion of variants in LD $\left(r^{2}>0.1\right)$ with the GWS coding alleles rs1229984 and rs2066702. Of these, only rs10516440 (AD conditional analyses: EU, $P=1.34 \times 10^{-3}$; AA, $P=0.013$; trans, $P=7.44 \times 10^{-5}$ ) was a significant multitissue expression quantitative trait locus in random effects analysis for $A D H 1 B$ (fixed effects test statistic $S_{\mathrm{FE}}=319.4$, heterogeneity test statistic $S_{\mathrm{Het}}=27.6$, combined $P=1.4 \times 10^{-76}$ ), ADH1A $\quad\left(S_{\mathrm{FE}}=139.4, \quad S_{\mathrm{Het}}=6.6, \quad\right.$ combined $\left.P=6.72 \times 10^{-33}\right)$, and $A D H 1 C\left(S_{\mathrm{FE}}=167.3, S_{\mathrm{Het}}=8.9\right.$, combined $\left.P=1.9 \times 10^{-39}\right)$. Rs10516440 is an LD proxy $\left(r^{2}>0.9\right)$ of rs6827898 (Table 2) in populations of European and African descent. These variants are both located in an intergenic region in the $A D H$ gene cluster between $A D H 1 C$ and $A D H 7$. In line with the fact that the protective coding alleles are associated with increased activity of the enzyme encoded by $A D H 1 B$, the major allele $\mathrm{rs} 10516440^{\star} \mathrm{A}$ was associated with increased $A D H 1 B$ expression and reduced $\mathrm{AD}$ risk. 
Table 1 | Descriptive statistics for cohorts in the meta-analysis of AD

\begin{tabular}{|c|c|c|c|c|c|c|c|c|c|c|c|}
\hline \multirow[t]{3}{*}{ Dataset } & \multirow[t]{3}{*}{ PMID } & \multirow{3}{*}{$\begin{array}{l}\text { Male } \\
(\%)\end{array}$} & \multirow{3}{*}{$\begin{array}{l}\text { Ages } \\
\text { (years) }\end{array}$} & \multicolumn{4}{|c|}{ EU } & \multicolumn{4}{|c|}{ AA } \\
\hline & & & & \multicolumn{2}{|c|}{$n$ total } & \multicolumn{2}{|c|}{$n$ unrelated } & \multicolumn{2}{|c|}{$n$ total } & \multicolumn{2}{|c|}{$n$ unrelated } \\
\hline & & & & Case & Control & Case & Control & Case & Control & Case & Control \\
\hline \multicolumn{12}{|c|}{ Case-control: logistic regression } \\
\hline $\begin{array}{l}\text { Comorbidity and Trauma } \\
\text { Study }\end{array}$ & 23303482 & $56 \%$ & $18-67$ & 572 & 817 & 572 & 817 & - & - & - & - \\
\hline $\begin{array}{l}\text { Christchurch Health and } \\
\text { Development Study }\end{array}$ & 23255320 & $48 \%$ & $16-30$ & 112 & 500 & 112 & 500 & - & - & - & - \\
\hline $\begin{array}{l}\text { COGA case-control cohort } \\
(\text { COGA-cc) }\end{array}$ & 20201924 & $54 \%$ & $18-79$ & 583 & 363 & 583 & 363 & - & - & - & - \\
\hline $\begin{array}{l}\text { Family Study of Cocaine } \\
\text { Dependence }\end{array}$ & 18243582 & $51 \%$ & $18-60$ & 266 & 174 & 266 & 174 & 255 & 241 & 255 & 241 \\
\hline $\begin{array}{l}\text { German Study of the } \\
\text { Genetics of Alcoholism } \\
\text { (GESGA) }\end{array}$ & 19581569 & $65 \%$ & $18-84$ & 1,314 & 2,142 & 1,314 & 2,142 & - & - & - & - \\
\hline $\begin{array}{l}\text { Gene-Environment } \\
\text { Development Initiative } \\
\text { (GEDI): Great Smoky } \\
\text { Mountains Study (GSMS) }\end{array}$ & 8956679 & $57 \%$ & $9-26$ & 42 & 565 & 42 & 565 & - & - & - & - \\
\hline $\begin{array}{l}\text { Center on Antisocial Drug } \\
\text { Dependence }\end{array}$ & 25637581 & $70 \%$ & $13-20$ & 400 & 577 & 400 & 577 & 51 & 51 & 51 & 51 \\
\hline $\begin{array}{l}\text { Phenomics and Genomics } \\
\text { Sample }\end{array}$ & 28371232 & $57 \%$ & $18-74$ & 37 & 523 & 37 & 523 & - & - & - & - \\
\hline $\begin{array}{l}\text { Collaborative Study on } \\
\text { the Genetics of Nicotine } \\
\text { Dependence (COGEND } \\
\text { Nico) }\end{array}$ & 17158188 & $34 \%$ & $25-82$ & 135 & 272 & 135 & 272 & 46 & 232 & 46 & 232 \\
\hline $\begin{array}{l}\text { COGEND Study of } \\
\text { Addiction: Genetics and } \\
\text { Environment (COGEND } \\
\text { SAGE) }\end{array}$ & 20202923 & $37 \%$ & $18-77$ & 311 & 225 & 311 & 225 & 104 & 103 & 104 & 103 \\
\hline Spit For Science & 24639683 & $36 \%$ & $>18$ & 252 & 1,863 & 252 & 1,863 & 74 & 841 & 74 & 841 \\
\hline NIAAA & $\mathrm{n} / \mathrm{a}$ & $67 \%$ & $>18$ & 442 & 206 & 442 & 206 & 404 & 110 & 404 & 110 \\
\hline $\begin{array}{l}\text { Mayo Clinic Center for the } \\
\text { Individual Treatment of } \\
\text { Alcohol Dependence }\end{array}$ & 25290263 & $55 \%$ & $\geq 18$ & 378 & 646 & 378 & 646 & - & - & - & - \\
\hline $\begin{array}{l}\text { Alcohol Dependence in } \\
\text { African Americans }\end{array}$ & $\mathrm{n} / \mathrm{a}$ & $57 \%$ & $18-69$ & - & - & - & - & 794 & 297 & 794 & 297 \\
\hline \multicolumn{12}{|c|}{ Family-based, twins, and siblings: generalized estimating equations } \\
\hline $\begin{array}{l}\text { Brisbane Longitudinal Twin } \\
\text { Study }\end{array}$ & 23187020 & $43 \%$ & $18-30$ & 60 & 938 & 51 & 546 & - & - & - & - \\
\hline $\begin{array}{l}\text { GEDI Virginia Twin Study } \\
\text { on Adolescent Behavioral } \\
\text { Development (VTSABD) }\end{array}$ & 9294370 & $38 \%$ & $8-32$ & 209 & 503 & 188 & 318 & - & - & - & - \\
\hline $\begin{array}{l}\text { Minnesota Center for Twin } \\
\text { and Family Research }\end{array}$ & 23942779 & $41 \%$ & $16-21$ & 609 & 2,100 & 553 & 1,274 & - & - & - & - \\
\hline $\begin{array}{l}\text { Center for Education and } \\
\text { Drug Abuse Research }\end{array}$ & 21514569 & $63 \%$ & $16-34$ & 59 & 200 & 54 & 152 & - & - & - & - \\
\hline Swedish Twin Registry & 23137839 & $47 \%$ & $40-83$ & 76 & 8,311 & 76 & 6,112 & - & - & - & - \\
\hline Yale-Penn & 24166409 & $58 \%$ & $16-79$ & 1,094 & 301 & 1,004 & 252 & - & - & - & - \\
\hline \multicolumn{12}{|c|}{ Family-based, large, or complex pedigrees: logistic mixed model } \\
\hline $\begin{array}{l}\text { COGA family cohort } \\
\text { (COGA-fam) }\end{array}$ & 23089632 & $45 \%$ & $12-88$ & 605 & 682 & 168 & 138 & - & - & - & - \\
\hline $\begin{array}{l}\text { Australian Alcohol and } \\
\text { Nicotine Studies }\end{array}$ & 21529783 & $45 \%$ & $18-82$ & 1,571 & 3,069 & 1,111 & 805 & - & - & - & - \\
\hline
\end{tabular}


Table 1 | Descriptive statistics for cohorts in the meta-analysis of AD (continued).

\begin{tabular}{|c|c|c|c|c|c|c|c|c|c|c|c|}
\hline \multirow[t]{3}{*}{ Dataset } & \multirow[t]{3}{*}{ PMID } & \multirow{3}{*}{$\begin{array}{l}\text { Male } \\
(\%)\end{array}$} & \multirow{3}{*}{$\begin{array}{l}\text { Ages } \\
\text { (years) }\end{array}$} & \multicolumn{4}{|c|}{ EU } & \multicolumn{4}{|c|}{ AA } \\
\hline & & & & \multicolumn{2}{|c|}{$n$ total } & \multicolumn{2}{|c|}{$n$ unrelated } & \multicolumn{2}{|c|}{$n$ total } & \multicolumn{2}{|c|}{$n$ unrelated } \\
\hline & & & & Case & Control & Case & Control & Case & Control & Case & Control \\
\hline $\begin{array}{l}\text { Irish Affected Sib Pair Study } \\
\text { of Alcohol Dependence }\end{array}$ & 15770118 & $50 \%$ & $17-84$ & 721 & 1,814 & 436 & 1,802 & - & - & - & - \\
\hline Yale-Penn & 24166409 & $51 \%$ & $16-79$ & - & - & - & - & 1,607 & 1,070 & 1,263 & 933 \\
\hline \multicolumn{12}{|l|}{ Summary statistics } \\
\hline $\begin{array}{l}\text { Netherlands Study of } \\
\text { Depression and Anxiety \& } \\
\text { Netherlands Twin Registry }\end{array}$ & 18197199 & $31 \%$ & $>18$ & 390 & 1,633 & 390 & 1,633 & - & - & - & - \\
\hline $\begin{array}{l}\text { Finnish Nicotine Addiction } \\
\text { Genetics Project (NAG-Fin) }\end{array}$ & 17436240 & $52 \%$ & $30-92$ & 439 & 1,137 & 439 & 1,137 & - & - & - & - \\
\hline FinnTwin12 & 17254406 & $47 \%$ & $20-27$ & 88 & 874 & 88 & 874 & - & - & - & - \\
\hline $\begin{array}{l}\text { National Longitudinal Study } \\
\text { of Adolescent to Adult } \\
\text { Health (Add Health) }\end{array}$ & 25378290 & $47 \%$ & $24-34$ & 768 & 2,981 & 768 & 2,981 & - & - & - & - \\
\hline Helsinki Birth Cohort Study & 16251536 & $43 \%$ & $56-70$ & 36 & 1,583 & 36 & 1,583 & - & - & - & - \\
\hline Total & & & & 11,569 & 34,999 & 10,206 & 28,480 & 3,335 & 2,945 & 2,991 & 2,808 \\
\hline
\end{tabular}

Overview of numbers of individuals with AD (cases) and controls from each cohort in the current analysis, including the number of genetically unrelated individuals. Cohorts are listed by study design and analysis method. Sample sizes are listed after quality control exclusions and stratified by ancestry group. PubMed identifiers (PMID) are listed for previous publications describing each cohort, along with the percentage of male samples and the age range in the cohort.

Associations with other GWS loci. We examined results for the eight independent variants associated at GWS levels with alcohol consumption in the UK Biobank ${ }^{17}$ (Supplementary Table 7). Among the UK Biobank findings, three of the four reported variants in the $A D H$ region of chromosome 4 (rs145452708, a proxy for rs1229984 with $D^{\prime}=1$, rs29001570 and rs35081954) were associated in the present study with $\mathrm{AD}$ ( $P$ values ranging from $3.5 \times 10^{-5}$ to $2.3 \times 10^{-10}$ ) with sign-concordant effects; the remaining variant was excluded from our analysis due to MAF $<0.01$. The UK Biobank lead variant in $K L B$, rs11940694, was nominally associated with AD $(P=0.0097)$, though this did not surpass multiple-testing correction for the eight GWS alcohol consumption loci. We saw little evidence $(P>0.2)$ for association of $\mathrm{AD}$ with the reported loci at GCKR and $C A D M 2$, which may be due to differences in power for the given effect size or because these genes exert an influence on liability to consume alcohol but not later problems. The locus on chromosome 18 showed limited regional association with $\mathrm{AD}$, but the index variant was not present in our analysis because it no longer appears in the 1000 Genomes Project Phase 3 reference panel ${ }^{21}$.

Polygenic risk score analyses. PRS based on our meta-analysis of $\mathrm{AD}$ were significantly predictive of $\mathrm{AD}$ outcomes in all three tested external cohorts. PRS derived from the unrelated EU GWAS predicted up to $0.51 \%$ of the variance in past month alcohol use disorder in the Avon Longitudinal Study of Parents and Children (ALSPAC; $P=0.0195$; Supplementary Fig. 10a) and up to $0.3 \%$ of problem drinking in Generation Scotland $\left(P=7.9 \times 10^{-6}\right.$; Supplementary Fig. 10b) as indexed by the CAGE (Cutting down, Annoyance by criticism, Guilty feelings, and Eye-openers) questionnaire. PRS derived from the unrelated AA GWAS predicted up to $1.7 \%$ of the variance in alcohol dependence in the COGA AAfGWAS cohort $\left(P=1.92 \times 10^{-7}\right.$; Supplementary Fig. $\left.10 \mathrm{c}\right)$.

Notably, PRS derived from the unrelated EU GWAS showed much weaker prediction (maximum $r^{2}=0.37 \%, \quad P=0.01$; Supplementary Fig. 10d) in the COGA AAfGWAS than the ancestrally matched AA GWAS-based PRS despite the much smaller discovery sample for AA. In addition, the AA GWAS-based AD PRS also still yielded significant variance explained after controlling for other genetic factors $\left(r^{2}=1.16 \%, P=2.5 \times 10^{-7}\right)$. Prediction of CAGE scores in Generation Scotland remained significant and showed minimal attenuation $\left(r^{2}=0.29 \%, P=1.0 \times 10^{-5}\right)$ after conditioning on PRS for alcohol consumption derived from UK Biobank results ${ }^{17}$. In COGA AAfGWAS, the AA PRS derived from our study continued to predict $1.6 \%$ of the variance in alcohol dependence after inclusion of rs2066702 genotype as a covariate, indicating independent polygenic effects beyond the lead $A D H 1 B$ variant (Supplementary Methods).

Power analysis. Power analyses indicated that the current metaanalysis is expected to have at least $41 \%$ power to detect very common variants (MAF $\geq 0.25$ ) with odds ratios $\geq 1.10$ at $P<5 \times 10^{-8}$ and $63 \%$ power for $P<1 \times 10^{-6}$ (Supplementary Fig. 11). Power at $P<1 \times 10^{-6}$ is relevant because only five loci reach that threshold in the current meta-analysis. Power is lower for less-common variants (MAF $\leq 0.05)$ even with odds ratios $\geq 1.20$ at $P<1 \times 10^{-6}(60 \%$ power) and $P<5 \times 10^{-8}$ (38\% power).

For perspective, power computations using the observed distribution of top effects for other large GWAS of polygenic traits suggest that we observed significantly fewer GWS loci for AD than would be expected if the loci had true effect sizes and allele frequencies similar to schizophrenia (expected: 25.4 loci, 95\% confidence interval: $21-30$ ) or obesity (expected: 8.9 loci, $95 \%$ confidence interval: 6-12), but not fewer than would be expected for effect sizes similar to major depression (Supplementary Methods and Supplementary Table 8).

\section{Discussion}

To our knowledge, this is the largest GWAS of rigorously defined $\mathrm{AD}$ to date, comprising 14,904 AD individuals and 37,944 controls. We identified known loci in $A D H 1 B$ that differed between EU and AA, as well as previously uncharacterized genetic correlations between $\mathrm{AD}$ and psychiatric disorders (for example, schizophrenia), tobacco and cannabis use, and social (for example, socioeconomic deprivation) and behavioral (for example, educational attainment) outcomes. Analyses also revealed a genetic distinction between GWAS results for alcohol consumption and AD. 
Table 2 | Top ten loci from the meta-analyses of AD by ancestry

\begin{tabular}{|c|c|c|c|c|c|c|c|c|c|c|c|c|c|c|}
\hline \multirow[t]{2}{*}{ SNP } & \multirow[t]{2}{*}{ Chr } & \multirow[t]{2}{*}{ BP } & \multirow[t]{2}{*}{ A1 } & \multirow[t]{2}{*}{ A2 } & \multirow[t]{2}{*}{ Gene } & \multicolumn{2}{|c|}{$\begin{array}{l}\text { A1 allele } \\
\text { frequency }\end{array}$} & \multicolumn{2}{|c|}{$\begin{array}{l}\text { INFO } \\
\text { score }\end{array}$} & \multicolumn{2}{|c|}{$\begin{array}{l}\text { Effect size } \\
\quad(O R)\end{array}$} & \multicolumn{3}{|c|}{$\begin{array}{c}\text { Discovery meta-analysis } \\
\qquad \text { value }\end{array}$} \\
\hline & & & & & & EU & AA & 1 & 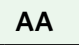 & U & 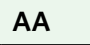 & U & AA & Trans \\
\hline \multicolumn{15}{|c|}{ Top clumped variants in transancestral meta-analysis $(14,904$ cases; 37,944 controls) } \\
\hline rs7644567^ & 3 & 29201672 & A & G & RBMS3 & 0.964 & 0.705 & 0.96 & 1.00 & - & 1.229 & $3.94 \times 10^{-4}$ & $6.64 \times 10^{-6}$ & $1.36 \times 10^{-8}$ \\
\hline rs2066702 & 4 & 100229017 & $A$ & G & $A D H 1 B$ & - & 0.215 & - & 0.99 & - & 0.731 & - & $2.21 \times 10^{-9}$ & $2.21 \times 10^{-9}$ \\
\hline rs1229984 & 4 & 100239319 & $T$ & C & $A D H 1 B$ & 0.040 & 0.014 & 0.90 & 0.91 & 0.486 & 0.912 & $9.79 \times 10^{-13}$ & $3.48 \times 10^{-1}$ & $2.18 \times 10^{-11}$ \\
\hline rs1789912 & 4 & 100263942 & $\mathrm{~T}$ & C & $A D$ & 0.418 & 0.132 & 1.00 & 1.02 & 1.106 & 1.211 & $1.98 \times 10^{-7}$ & $1.32 \times 10^{-3}$ & $1.47 \times 10^{-9}$ \\
\hline rs6827898 & 4 & 100295863 & A & G & egion) & 0.123 & 0.112 & 0.96 & 0.94 & & 70 & & $9.31 \times 10^{-4}$ & $2.97 \times 10^{-9}$ \\
\hline rs894368 & 4 & 10030 & A & C & egion) & 0.309 & 86 & & & & 81 & & & $3.30 \times 10^{-7}$ \\
\hline 618 & 7 & 68 & A & G & - & - & & - & & - & & - & & $6.30 \times 10^{-7}$ \\
\hline s116338421 & 8 & 145761 & C & G & & - & 0.172 & - & 0.91 & - & 0.755 & - & & \\
\hline 79171978 & 12 & 17798824 & C & G & - & 0.099 & 0.027 & 0.9 & 0.99 & 1.201 & 1.016 & $5.47 \times 10^{-8}$ & $8.18 \times 10^{-1}$ & $5.98 \times 10^{-7}$ \\
\hline s8017647 & 14 & 32456358 & $T$ & C & - & 0.792 & 0.565 & 1.00 & 0.99 & 0.901 & 0.923 & $8.05 \times 10^{-6}$ & & $<10^{-6}$ \\
\hline \multicolumn{15}{|c|}{ Top clumped variants in African ancestry meta-analysis ( 3,335 cases; 2,945 controls) } \\
\hline s5781337 & 1 & 223883425 & CA & C & - & 0.263 & 0.212 & 0.98 & 0.93 & 1.007 & 0.664 & & $1.62 \times 10^{-7}$ & 6.5 \\
\hline s143258048 & 3 & 75982870 & A & $A C$ & $\mathrm{ROBO} 2$ & - & 0.028 & - & 0.88 & - & 0.490 & - & $1.86 \times 10^{-6}$ & - \\
\hline 3857224 & 4 & 100129685 & $\mathrm{~T}$ & C & $A D H 6$ & 0.315 & 0.585 & 0.99 & 1.00 & 0.970 & 0.814 & $2.40 \times 10^{-1}$ & $5.86 \times 10^{-7}$ & $2.36 \times 10^{-3}$ \\
\hline rs2066702 & 4 & 100229017 & A & G & $A D H 1 B$ & - & 0.215 & - & 0.99 & - & 0.731 & - & $2.21 \times 10^{-9}$ & $2.21 \times 10^{-9}$ \\
\hline 52461618 & 7 & 68667233 & A & G & - & - & 0.088 & - & 0.98 & - & 0.669 & - & $6.30 \times 10^{-7}$ & $6.30 \times 10^{-7}$ \\
\hline 5116338421 & 8 & 1457 & C & G & $A R$ & - & 0.172 & - & 0 & - & 55 & - & $4.86 \times 10^{-7}$ & $4.86 \times 10^{-7}$ \\
\hline s79016499 & 11 & 93010988 & $\mathrm{~T}$ & $C$ & - & - & 0.066 & - & 0.93 & - & 1.128 & - & $1.36 \times 10^{-6}$ & - \\
\hline s10784244 & 12 & 62035165 & G & A & - & 0.153 & 0.484 & 1.00 & 1.00 & 1.041 & 1.226 & $6.26 \times 10^{-2}$ & $1.04 \times 10^{-6}$ & $2.49 \times 10^{-4}$ \\
\hline s17199739 & 16 & 25444288 & G & A & - & 0.176 & 0.096 & 0.99 & 0.96 & 0.994 & 0.693 & $4.25 \times 10^{-1}$ & $1.11 \times 10^{-6}$ & $8.66 \times 10^{-3}$ \\
\hline rs740793 & 17 & 3846353 & G & $A$ & ATP2A3 & 0.453 & 0.350 & 0.97 & 0.97 & 0.996 & 1.370 & $4.66 \times 10^{-1}$ & $1.48 \times 10^{-6}$ & $3.44 \times 10^{-1}$ \\
\hline \multicolumn{15}{|c|}{ Top clumped variants in European ancestry meta-analysis ( 11,569 cases; 34,999 controls) } \\
\hline rs1229984 & 4 & 100239319 & $\mathrm{~T}$ & $\mathrm{C}$ & $A D H 1 B$ & 0.040 & 0.014 & 0.90 & 0.91 & 0.486 & 0.912 & $9.79 \times 10^{-13}$ & $3.48 \times 10^{-1}$ & $2.18 \times 10^{-11}$ \\
\hline rs3811802 & 4 & 100244221 & G & A & $A D H 1 B$ & 0.454 & 0.529 & 0.96 & 0.96 & 1.162 & 0.914 & $2.40 \times 10^{-8}$ & $2.19 \times 10^{-2}$ & $1.22 \times 10^{-4}$ \\
\hline rs113659074 & 4 & 100252308 & $\mathrm{~T}$ & G & $A D H 1 B$ & 0.068 & 0.093 & 0.98 & 0.95 & 0.800 & 1.166 & $1.54 \times 10^{-6}$ & $6.63 \times 10^{-2}$ & $2.99 \times 10^{-4}$ \\
\hline rs1229863 & 4 & 100252386 & A & $T$ & $A D H 1 B$ & 0.174 & 0.038 & 0.99 & 0.99 & 1.145 & 1.254 & $7.80 \times 10^{-7}$ & $4.26 \times 10^{-2}$ & $9.28 \times 10^{-8}$ \\
\hline rs1154445 & 4 & 100288521 & G & $\mathrm{T}$ & (ADH region) & 0.425 & 0.134 & 0.97 & 0.99 & 1.137 & 1.211 & $1.80 \times 10^{-7}$ & $2.63 \times 10^{-2}$ & $1.48 \times 10^{-8}$ \\
\hline rs6827898 & 4 & 100295863 & A & G & (ADH region) & 0.123 & 0.112 & 0.96 & 0.94 & 1.145 & 1.270 & $5.21 \times 10^{-7}$ & $9.31 \times 10^{-4}$ & $2.97 \times 10^{-9}$ \\
\hline rs894368 & 4 & 100309313 & A & C & (ADH region) & 0.309 & 0.386 & 0.99 & 0.96 & 0.887 & 0.981 & $1.93 \times 10^{-8}$ & $9.73 \times 10^{-1}$ & $3.30 \times 10^{-7}$ \\
\hline rs79171978 & 12 & 17798824 & C & G & - & 0.099 & 0.027 & 0.99 & 0.99 & 1.201 & 1.016 & $5.47 \times 10^{-8}$ & $8.18 \times 10^{-1}$ & $5.98 \times 10^{-7}$ \\
\hline rs4388946 & 12 & 17935154 & C & $A$ & - & 0.240 & 0.297 & 0.99 & 0.98 & 1.137 & 0.950 & $7.14 \times 10^{-7}$ & $1.87 \times 10^{-1}$ & $7.05 \times 10^{-5}$ \\
\hline rs34929220 & 15 & 69769635 & $\mathrm{~T}$ & C & DRAIC & 0.690 & 0.937 & 0.90 & 0.94 & 0.893 & 1.028 & $1.02 \times 10^{-6}$ & $8.38 \times 10^{-1}$ & $7.38 \times 10^{-6}$ \\
\hline
\end{tabular}

Top ten nominally independent variants from the discovery transancestral (trans.; $\left.n_{\text {case }}=14,904 ; n_{\text {control }}=37,944\right)$ meta-analysis and the discovery meta-analyses in AA ( $\left.n_{\text {case }}=3,335 ; n_{\text {control }}=2,945\right)$ and EU $\left(n_{\text {case }}=11,569 ; n_{\text {control }}=34,999\right)$ ancestry cohorts, respectively. Independent variants are identified based on clumping for LD (pairwise $\left.r^{2}<0.1\right)$ in 1000 Genomes Project Phase 3 data ${ }^{2}$. EU results are clumped using European ancestry reference samples, AA results are clumped using African ancestry reference samples from the American Southwest, and transancestral results are clumped using merged European and African ancestry reference samples. $P$ values and allele frequencies are reported from two-tailed tests of association with $A D$ in fixed-effects meta-analyses weighted by effective sample size. Bold $P$ values indicate GWS after correction for multiple testing within the analysis $\left(P<5 \times 10^{-8}\right)$. Odds ratios (OR) and INFO scores are reported from the meta-analyses of the subset of unrelated individuals within each ancestry. Variants are sorted by chromosome (Chr) and base pair (BP) position for genome build hg19, with genes annotated by Ensembl VEP49. Allele frequency and OR are given with respect to allele 1 (A1). SNPs included in the transancestral meta-analysis were not conditioned on being analyzed in both the EU and AA analyses. For instance, a SNP of strong effect in one group may not be sufficiently common or well-imputed for analysis in the other ancestral group (for example, rs2066702 is not found in non-African populations but is among the top ten in the transancestral analysis due to strong effects in the AA group). For rs 7644567 (denoted with *), the SNP did not pass quality control in a sufficient number of cohorts to meet the minimum sample-size requirement for inclusion in the EU-only analyses-it is only represented among EU cohorts by summary statistics from two Finnish cohorts-but allele frequency, INFO score, and meta-analyzed P values from the Finnish summary statistics are reported since they contribute to the transancestral meta-analysis.

Although larger sample sizes can be amassed by focusing on quantitative measures of consumption, only the upper tail is relevant to $\mathrm{AD}$ (as a medical diagnosis), and even that does not capture other aspects of disordered drinking (for example, loss of control, withdrawal) directly. Conversely, cases derived from electronic medical records (for example, International Classification of Diseases (ICD) codes) result in a high rate of false negatives, while self-screening instruments (for example, AUDIT scores) are best suited to analyses of disordered drinking when a sufficiently high threshold or score cutoff is applied to focus on severity. Our study has the advantage of greater diagnostic precision via use of semistructured interviews to diagnose AD systematically in a majority of the constituent studies and therefore greater interpretability in the context of clinically important AD.

The GWS SNPs reaffirm the importance of functional variants affecting alcohol metabolism to the risk of $\mathrm{AD}$. The top association in $A D H 1 B$, rs1229984, is a missense variant that is amongst the most widely studied in relation to alcohol use, misuse, and 


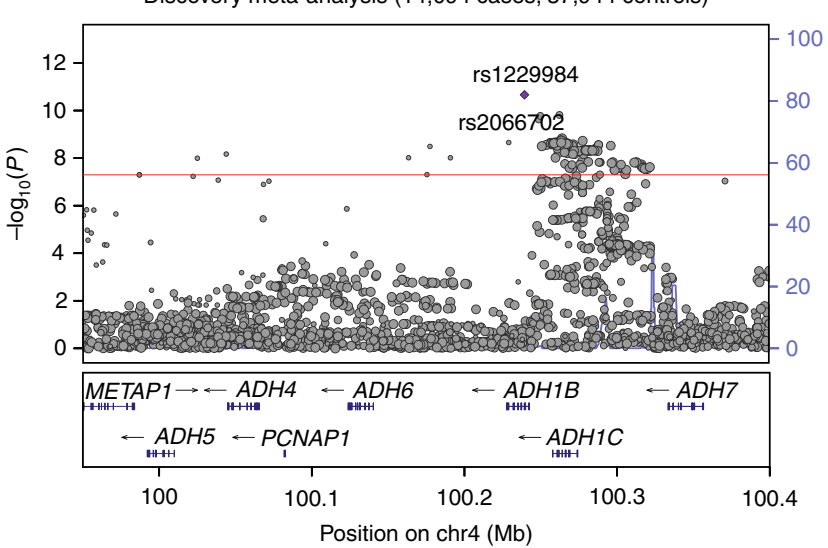

b

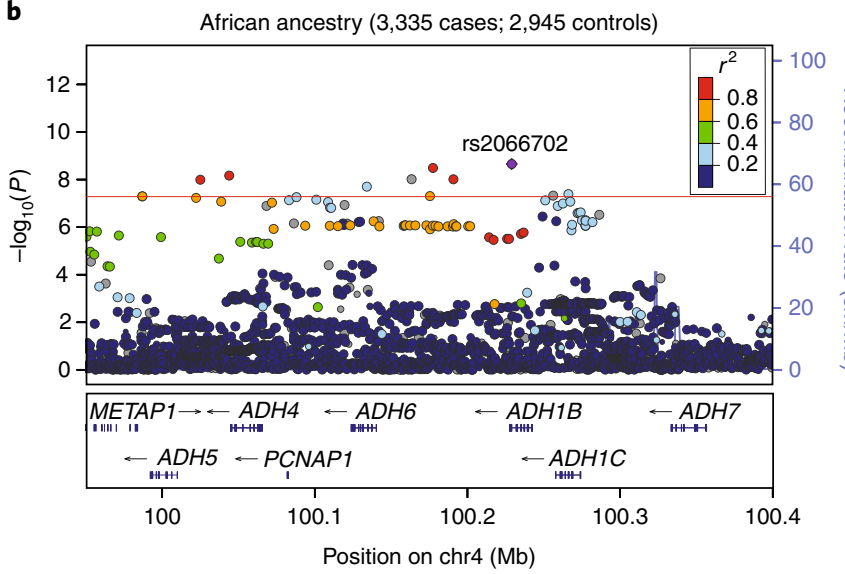

c

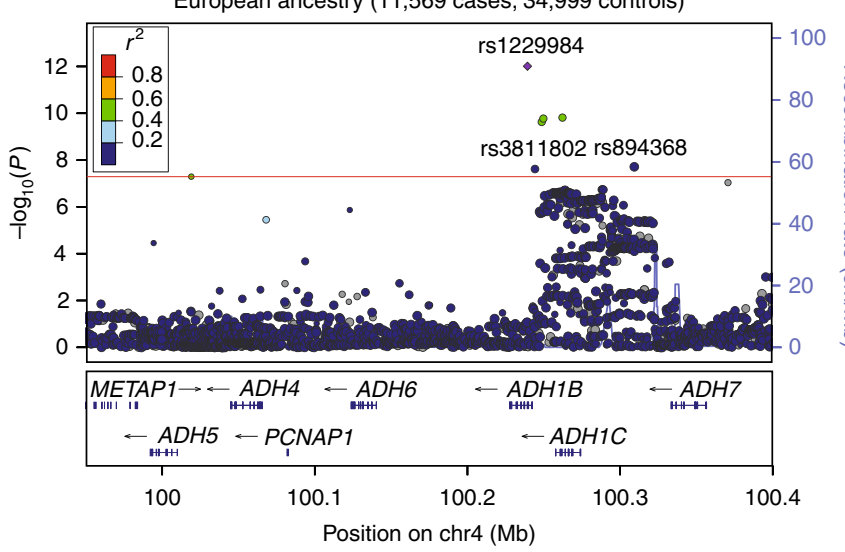

Fig. 2 | Regional plots for the ADH1B locus (rs1229984) in the transancestral discovery, AA, and EU meta-analyses. a-c, Results of fixed effects metaanalysis with effective sample-size weights for the $A D H 1 B$ locus in (a) all cohorts $\left(n_{\text {case }}=14,904 ; n_{\text {control }}=37,944\right)$; (b) AA cohorts $\left(n_{\text {case }}=3,335\right.$; $\left.n_{\text {control }}=2,945\right)$; and (c) EU cohorts $\left(n_{\text {case }}=11,569 ; n_{\text {control }}=34,999\right)$. Red reference line indicates the GWS threshold after correction for multiple testing within each analysis $\left(P<5 \times 10^{-8}\right)$. Within ancestry, colored points reflect the degree of LD (pairwise $r^{2}$ ) to the index variant (purple diamond) in 1000 Genomes Project reference data ${ }^{21}$ for individuals of (b) African or (c) European ancestry, respectively. LD structures in the two ancestries differ, so for the transancestral sample (a), LD is not given, indicted by gray points. Two-tailed tests used for all analyses. Chr4, chromosome 4.

dependence $^{8-10}$. The resulting amino acid substitution (Arg48His) increases the rate at which alcohol dehydrogenase $1 \mathrm{~B}$ oxidizes ethanol to acetaldehyde ${ }^{8}$. Studies on Asian populations in which the derived allele is common demonstrated strong protection against the development of $\mathrm{AD}^{8,9,13}$. In $\mathrm{EU}$ and $\mathrm{AA}$, the protective allele is present at much lower frequencies $(\mathrm{EU}, \mathrm{MAF}=0-4 \%$; $\mathrm{AA}$, MAF $<1 \%$ ); nevertheless, recent large-scale studies have shown an association between this locus and alcohol consumption and problems at GWS levels in EU with similar effect size ${ }^{8-10}$. The lead variant in AA cohorts, rs2066702 (Arg370Cys), is another functional missense variant in $A D H 1 B$, and it also encodes an enzyme with an increased rate of ethanol oxidation ${ }^{8}$. The allele encoding Cys 370 is common in AA, but rare in other populations ${ }^{8}$. Our results clearly show that these two different functional SNPs in $A D H 1 B$ both affect risk for alcoholism, with their relative importance dependent upon allele frequency in the population studied. There is a suggestion of additional independent effects in the chromosome 4 region, but larger studies will be needed to evaluate this.

The only other locus to reach significance was rs7644567 on chromosome 3, primarily driven by AA cohorts. The locus failed to replicate in two small, independent AA samples, and in the only European cohort with even a modest allele frequency (FINRISK), the effect was in the opposite direction. There have also been discussions about whether the standard GWAS significance threshold should be applied to the more genetically diverse African-ancestry cohorts $^{22,23}$ and about the possibility of confounding from nonlinear relationships between phenotypes and ancestry-informative markers like rs7644567 in admixed samples ${ }^{24}$, all of which increase our skepticism regarding this finding. There is, therefore, insufficient evidence at this time to conclude that rs7644567 is associated with alcohol dependence. Analyses of much larger samples of African ancestry will be needed to resolve this.

Despite limited SNP-level findings, there is significant evidence for polygenic effects of common variants in both EU and AA cohorts. The estimated $h_{g}^{2}=0.09$ for AD in EU is only modestly lower than those recently reported for alcohol consumption $\left(h_{g}^{2}=0.13\right)^{17}$ and AUDIT scores $\left(h_{g}^{2}=0.12\right)^{18}$, and it is comparable to estimates derived for cigarettes-per-day ${ }^{25}$. Our $h_{g}^{2}$ estimate is lower than that of a prior report ${ }^{7}$, likely reflecting a combination of differences in estimation method (genomic relatedness matrix restricted maximum likelihood (GREML) versus LDSR) and greater heterogeneity in ascertainment strategy across samples in the current study (see refs ${ }^{26-28}$ ). The latter is especially relevant in comparing $h_{g}^{2}$ from that prior single cohort to our meta-analysis, which included cohorts with a wide range of ages at ascertainment, cultural environments, and ascertainment strategies, including enrichment for other substance use disorders. Similarly to other psychiatric disorders (for example, schizophrenia), a much larger sample size will potentially aid in overcoming across-sample heterogeneity and will capture a greater proportion of genetic variance.

Comparing our GWAS to recent GWAS of alcohol consumption measures suggests that the liability underlying normative patterns of alcohol intake and AD are only partially overlapping. Genome-wide genetic correlations were significantly $<1$ with log-scaled alcohol consumption by participants in AlcGen and CHARGE+ Consortia cohorts $^{16}\left(r_{g}=0.695\right.$, s.e. $=0.15$, one-tailed $P=0.024$ for $\left.r_{g}<1\right)$ and in the UK Biobank ${ }^{17}\left(r_{g}=0.371\right.$, s.e. $=0.092$, one-tailed $P=3.2 \times 10^{-12}$ for $r_{g}<1$ ). We also observed only partial replication of the eight loci significantly associated with consumption in the UK Biobank, with strongest results from SNPs in the $A D H$ region, including a proxy for rs1229984. In addition, there was no significant correlation with GWAS of log-scaled AUDIT scores in 23andMe participants ${ }^{18}$ $\left(r_{g}=0.076\right.$, s.e. $=0.171$, two-tailed $\left.P=0.656\right)$. Subsequent analyses suggest these estimates are sensitive to sample characteristics, with somewhat higher genetic correlations reported in analysis of alcohol consumption in the full UK Biobank ${ }^{29}\left(r_{g}=0.75\right)$ and of AUDIT in combined data from 23andMe participants and UK Biobank ${ }^{30}$ $\left(r_{g}=0.39\right)$. Notably, initial UK Biobank data includes a subset of participants recruited for a study of smoking and lung function in the 
Cannabis initiation $\left(P=3 \times 10^{-4}\right)$
Smoking initiation $\left(P=1 \times 10^{-7}\right)$

Nicotine dependence $\left(P=2 \times 10^{-4}\right)$

Smoking cessation $\left(P=2 \times 10^{-5}\right)$

Cigarettes per day $\left(P=6 \times 10^{-2}\right)$

Alcohol consumption (Schumann) $\left(P=7 \times 10^{-6}\right)$

Alcohol consumption (Clarke) $\left(P=5 \times 10^{-5}\right)$

Alcohol use (AUDIT) scores $\left(P=7 \times 10^{-1}\right)$

Depressive symptoms $\left(P=3 \times 10^{-7}\right)$

Major depressive disorder $\left(P=3 \times 10^{-11}\right)$

ADHD $\left(P=4 \times 10^{-6}\right)$

PGC cross-disorder $\left(P=2 \times 10^{-4}\right)$

Schizophrenia $\left(P=3 \times 10^{-11}\right)$

Bipolar disorder $\left(P=5 \times 10^{-2}\right)$

Alzheimer's disease $\left(P=5 \times 10^{-1}\right)$

Anorexia nervosa $\left(P=8 \times 10^{-1}\right)$

Autism spectrum disorder $\left(P=5 \times 10^{-1}\right)$

Obsessive-compulsive disorder $\left(P=4 \times 10^{-2}\right)$

Neuroticism $\left(P=2 \times 10^{-5}\right)$

Subjective well being $\left(P=3 \times 10^{-6}\right)$

Educational attainment $\left(P=1 \times 10^{-12}\right)$

Townsend deprivation $\left(P=7 \times 10^{-11}\right)$

Delay discounting $\left(P=6 \times 10^{-3}\right)$

Risk taking $\left(P=7 \times 10^{-3}\right)$

Age of first birth $\left(P=2 \times 10^{-9}\right)$

Number of children $\left(P=5 \times 10^{-4}\right)$

Parents' age at death $\left(P=3 \times 10^{-2}\right)$

Coronary artery disease $\left(P=1 \times 10^{-2}\right)$

Type 2 Diabetes $\left(P=7 \times 10^{-1}\right)$

Plasma GGT $\left(P=3 \times 10^{-3}\right)$

Plasma ALT $\left(P=5 \times 10^{-2}\right)$

Plasma AST $\left(P=2 \times 10^{-1}\right)$

Plasma ALP $\left(P=3 \times 10^{-1}\right)$

$\operatorname{HbA} 1 \mathrm{C}\left(P=6 \times 10^{-2}\right)$

Serum creatinine $\left(P=2 \times 10^{-1}\right)$

Heart rate $\left(P=3 \times 10^{-1}\right)$

HDL cholesterol $\left(P=3 \times 10^{-1}\right)$

Leptin $(P=1)$

Intracranial volume $(P=1)$

Caudate volume $\left(P=3 \times 10^{-1}\right)$

Putamen volume $\left(P=6 \times 10^{-2}\right)$

Height $\left(P=9 \times 10^{-1}\right)$

Body mass index $\left(P=2 \times 10^{-1}\right)$

Chronotype $\left(P=5 \times 10^{-1}\right)$

Sleep duration $\left(P=7 \times 10^{-1}\right)$

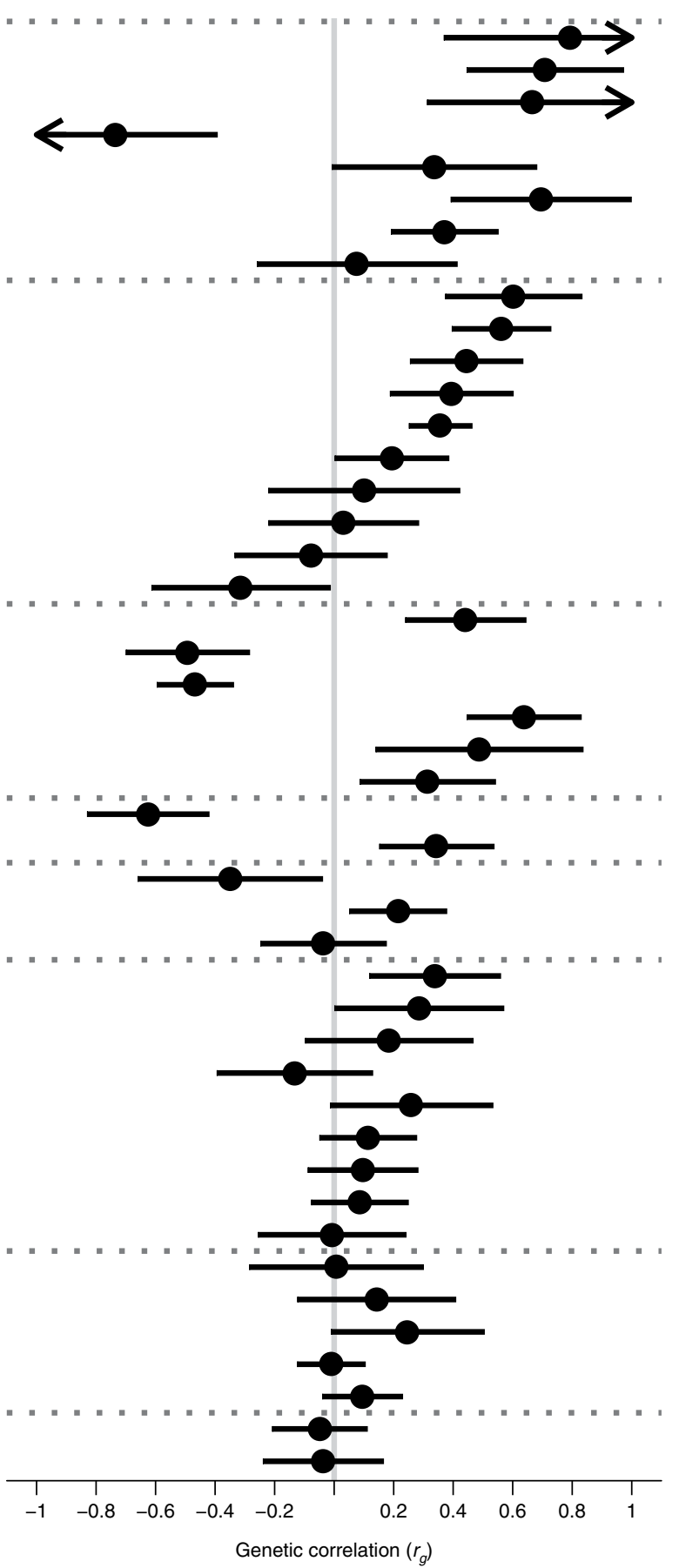

Fig. 3 | Genetic correlations between 45 traits and alcohol dependence in Europeans. Genetic correlation results from LDSR with the meta-analysis of AD in unrelated EU individuals ( $n_{\text {case }}=10,206 ; n_{\text {control }}=28,480$ ). After Bonferroni correction, significant correlations were observed with 17 traits and disorders $\left(P<1.1 \times 10^{-3}\right.$; bold), with nominally significant results for eight additional traits and disorders $(P<0.05$; italics $)$ based on two-tailed tests of the estimated genetic correlation with block jackknife standard errors. Error bars indicate $95 \%$ confidence intervals; arrows indicate intervals extending above 1 or below -1. Vertical gray reference line corresponds to the null hypothesis of no genetic correlation with AD. Phenotypes are organized by research domain. ADHD, attention deficit-hyperactivity disorder; HDL, high-density lipoprotein; HbA1c, hemoglobin A1c; GGT, gamma-glutamyl transferase; AST, aspartate aminotransferase; ALT, alanine aminotransferase; ALP, alkaline phosphatase.

first analysis ${ }^{17}$, which may have resulted in collider bias ${ }^{31}$ and contributed to the initial lower genetic correlation.

One key factor in interpreting the differences between these traits and $\mathrm{AD}$ is that the distribution of consumption levels and AUDIT scores can be highly skewed in population samples, with most individuals at the low (nonpathological) end of the spectrum. This effect may be especially pronounced among the older, healthy volunteers of the UK Biobank cohort ${ }^{32}$ and in the 23andMe cohort, which is more educated and has higher socioeconomic status than the general US population ${ }^{18}$. We hypothesize that the variants that affect consumption at lower levels may differ substantively from those that affect very high levels of consumption in alcohol dependent individuals, who are also characterized by loss of control over intake ${ }^{33}$. This appears to be the case in studies that used specific cut-offs to 
harmonize AUDIT scores with AD data ${ }^{30,34}$. The larger of these studies $^{30}$ reports that the genetic correlation between AD and AUDIT scores is maximized at an AUDIT cutoff $\geq 20$ (with controls defined as those scoring $\left.\leq 4 ; r_{g}=0.90\right)$. Notably, that study also found that a score reflecting items related to problem drinking (AUDIT-P) resulted in a stronger genetic correlation $\left(r_{g}=0.64\right)$ than a score related to alcohol consumption alone $\left(r_{g}=0.33\right)$. The strong genetic correlation of $\mathrm{AD}$ with lower educational attainment and lower socioeconomic status (i.e., higher Townsend deprivation), in contrast to positive genetic correlations of education with consumption ${ }^{17}$ and AUDIT scores related to consumption ${ }^{30}$, further underscore this distinction between normative or habitual levels of alcohol intake and diagnosed $\mathrm{AD}$, at least in the respective populations studied.

The current analysis identified robust genetic correlation of $\mathrm{AD}$ with a broad variety of psychiatric outcomes. This correlation was strongest for aspects of negative mood, including neuroticism and major depression, as also seen in twin studies ${ }^{35,36}$ and through recent specific molecular evidence for pleiotropy ${ }^{37,38}$. Taken together with evidence from other recent genomic studies ${ }^{37}$, and with null correlations for other GWAS of alcohol consumption but not for measures of problem drinking (for example, AUDIT-P), these findings suggest that major depression may primarily share genetic liability with alcohol use at pathological levels.

$\mathrm{AD}$ was also strongly genetically correlated with poor educational and socioeconomic outcomes and marginally correlated with measures of risk-taking. Nominally significant genetic correlations with delay discounting (i.e., favoring immediate rewards) and risktaking, and the strong genetic correlation of $\mathrm{AD}$ with attention deficit-hyperactivity disorder, cigarette smoking, and cannabis use, may similarly reflect a shared genetic factor for risk-taking and reduced impulse control. Common genetic liability to early, risky behaviors is characteristic of both $\mathrm{AD}^{39}$ and age of first birth ${ }^{40}$. The observed negative genetic correlation with age of first birth is consistent both with risk-taking and with the significant genetic correlations of $\mathrm{AD}$ with lower socioeconomic status, as indexed by higher neighborhood Townsend deprivation score and lower educational attainment. Lower socioeconomic status is correlated with both $\mathrm{AD}^{41}$ and age of first birth ${ }^{42}$, and the current study suggests that shared genetic liabilities may be one potential mechanism for their observed relationship. However, the question of whether these genetic correlations represent causal processes, horizontal pleiotropy, or the impact of unmeasured confounders should be explored in the future ${ }^{43}$.

Lower genetic correlations were observed for most biomedical and anthropometric outcomes. Liver enzymes GGT and ALT, once proposed as possible biomarkers for alcohol abuse ${ }^{44}$, showed only nominal evidence for genetic correlation with $\mathrm{AD}$, and neither survived multiple-testing correction. Notably, we did not find any association between $\mathrm{AD}$ and body-mass index (BMI). Negative genetic correlations with BMI were previously reported for both alcohol consumption ${ }^{17}$ and AUDIT scores ${ }^{18}$, but there is prior evidence that BMI has differing underlying genetic architectures in the context of $\mathrm{AD}$ and outside of that context $\mathrm{t}^{45}$. The negative genetic correlations observed in those studies are consistent with studies of light to moderate drinking, which is also associated with healthier lifestyle behaviors, while heavy and problematic drinking is typically associated with weight gain ${ }^{46}$.

This study benefited from precision in diagnostic assessment of $\mathrm{AD}$, known alcohol exposure in a majority of the controls, and careful quality control that excluded overlap of individuals between studies. Despite these strengths, our sample size was insufficient to identify additional GWS loci robustly. Power analyses indicate that additional SNPs associated with AD are likely to have small effect sizes, smaller than schizophrenia ${ }^{47}$ and more consistent with more common psychiatric disorders (for example, major depression ${ }^{48}$ ). This supports the pressing need for collection of large numbers of well-characterized cases and controls. The differences between our results and the study of AUDIT scores ${ }^{18}$ highlight that ascertainment and trait definition are critically important and must be taken into account. Careful study of how screening tools, such as the AUDIT, correlate with genetic liability to $\mathrm{AD}$ (as defined by DSM-IV or similar) could substantially boost sample sizes for future AD GWAS. There is also a continued need to characterize the genetic architecture of $\mathrm{AD}$ in non-EU populations.

We show a previously unreported genetic distinction between drinking in the pathological range (AD) and habitual drinking that does not cross the threshold into pathology or dependence and does not capture behavioral aspects of disordered drinking. Larger future samples will allow us to uncover additional pleiotropy between pathological and nonpathological alcohol use, as well as between $\mathrm{AD}$ and other neuropsychiatric disorders.

\section{Online content}

Any methods, additional references, Nature Research reporting summaries, source data, statements of data availability and associated accession codes are available at https://doi.org/10.1038/ s41593-018-0275-1.

Received: 22 January 2018; Accepted: 12 October 2018; Published online: 26 November 2018

\section{References}

1. World Health Organization. Global Status Report on Alcohol and Health, 2014 (WHO Press, Geneva, Switzerland, 2014).

2. American Psychiatric Association. Diagnostic and Statistical Manual of Mental Disorders, Fourth Edition, Text Revision (APA Publishing, Washington DC, USA, 2000).

3. Hasin, D. S., Stinson, F. S., Ogburn, E. \& Grant, B. F. Prevalence, correlates, disability, and comorbidity of DSM-IV alcohol abuse and dependence in the United States: results from the National Epidemiologic Survey on Alcohol and Related Conditions. Arch. Gen. Psychiatry 64, 830-842 (2007).

4. Dawson, D. A., Grant, B. F., Stinson, F. S. \& Chou, P. S. Estimating the effect of help-seeking on achieving recovery from alcohol dependence. Addiction 101, 824-834 (2006)

5. Verhulst, B., Neale, M. C. \& Kendler, K. S. The heritability of alcohol use disorders: a meta-analysis of twin and adoption studies. Psychol. Med. 45, 1061-1072 (2015)

6. Hart, A. B. \& Kranzler, H. R. Alcohol dependence genetics: lessons learned from genome-wide association studies (GWAS) and post-GWAS analyses. Alcohol. Clin. Exp. Res. 39, 1312-1327 (2015).

7. Palmer, R. H. C. et al. Shared additive genetic influences on DSM-IV criteria for alcohol dependence in subjects of European ancestry. Addiction 110, 1922-1931 (2015).

8. Hurley, T. D. \& Edenberg, H. J. Genes encoding enzymes involved in ethanol metabolism. Alcohol Res. 34, 339-344 (2012).

9. Li, D., Zhao, H. \& Gelernter, J. Strong association of the alcohol dehydrogenase $1 \mathrm{~B}$ gene $(A D H 1 B)$ with alcohol dependence and alcoholinduced medical diseases. Biol. Psychiatry 70, 504-512 (2011).

10. Bierut, L. J. et al. $A D H 1 B$ is associated with alcohol dependence and alcohol consumption in populations of European and African ancestry. Mol. Psychiatry 17, 445-450 (2012).

11. Frank, J. et al. Genome-wide significant association between alcohol dependence and a variant in the $A D H$ gene cluster. Addict. Biol. 17, 171-180 (2012).

12. Li, D., Zhao, H. \& Gelernter, J. Strong protective effect of the aldehyde dehydrogenase gene $(A L D H 2)$ 504lys $\left({ }^{\star} 2\right)$ allele against alcoholism and alcohol-induced medical diseases in Asians. Hum. Genet. 131, 725-737 (2012).

13. Luczak, S. E., Glatt, S. J. \& Wall, T. L. Meta-analyses of $A L D H 2$ and $A D H 1 B$ with alcohol dependence in Asians. Psychol. Bull. 132, 607-621 (2006).

14. Sullivan, P. F. et al. Psychiatric genomics: an update and an agenda. Am. J. Psychiatry 175, 15-27 (2018).

15. de Leeuw, C. A., Mooij, J. M., Heskes, T. \& Posthuma, D. MAGMA: generalized gene-set analysis of GWAS data. PLoS Comput. Biol. 11, e1004219 (2015).

16. Schumann, G. et al. $K L B$ is associated with alcohol drinking, and its gene product $\beta$-Klotho is necessary for FGF21 regulation of alcohol preference. Proc. Natl. Acad. Sci. USA 113, 14372-14377 (2016).

17. Clarke, T.-K. et al. Genome-wide association study of alcohol consumption and genetic overlap with other health-related traits in UK Biobank ( $\mathrm{N}=112$ 117). Mol. Psychiatry 22, 1376-1384 (2017).

18. Sanchez-Roige, S. et al. Genome-wide association study of alcohol use disorder identification test (AUDIT) scores in 20328 research participants of European ancestry. Addict. Biol. https://doi.org/10.1111/adb.12574 (2017). 
19. Sanchez-Roige, S. et al. Genome-wide association study of delay discounting in 23,217 adult research participants of European ancestry. Nat. Neurosci. 21, 16-18 (2018).

20. Lonsdale, J. et al. GTEx Consortium. The Genotype-Tissue Expression (GTEx) project. Nat. Genet. 45, 580-585 (2013).

21. Auton, A. et al. The 1000 Genomes Project Consortium. A global reference for human genetic variation. Nature 526, 68-74 (2015).

22. Dudbridge, F. \& Gusnanto, A. Estimation of significance thresholds for genomewide association scans. Genet. Epidemiol. 32, 227-234 (2008).

23. Pe'er, I., Yelensky, R., Altshuler, D. \& Daly, M. J. Estimation of the multiple testing burden for genomewide association studies of nearly all common variants. Genet. Epidemiol. 32, 381-385 (2008).

24. Derks, E. M., Zwinderman, A. H. \& Gamazon, E. R. The relation between inflation in Type-I and Type-II error rate and population divergence in genome-wide association analysis of multi-ethnic populations. Behav. Genet. 47, 360-368 (2017).

25. Zheng, J. et al. LD Hub: a centralized database and web interface to perform LD score regression that maximizes the potential of summary level GWAS data for SNP heritability and genetic correlation analysis. Bioinformatics 33, 272-279 (2017).

26. Bulik-Sullivan, B. et al. An atlas of genetic correlations across human diseases and traits. Nat. Genet. 47, 1236-1241 (2015).

27. Evans, L. M. et al. Comparison of methods that use whole genome data to estimate the heritability and genetic architecture of complex traits. Nat. Genet 50, 737-745 (2018)

28. Wray, N. R. \& Maier, R. Genetic basis of complex genetic disease: the contribution of disease heterogeneity to missing heritability. Curr. Epidemiol. Rep. 1, 220-227 (2014)

29. Polimanti, R. et al. Evidence of causal effect of major depressive disorder on alcohol dependence: findings from the Psychiatric Genomics Consortium. Preprint at bioRxiv https://doi.org/10.1101/412098 (2018).

30. Sanchez-Roige, S. et al. Genome-wide association study meta-analysis of the alcohol use disorder identification test (AUDIT) in two population-based cohorts $(\mathrm{N}=141,958)$. Preprint at bioRxiv https://doi.org/10.1101/275917 (2018).

31. Munafò, M. R., Tilling, K., Taylor, A. E., Evans, D. M. \& Davey Smith, G. Collider scope: when selection bias can substantially influence observed associations. Int. J. Epidemiol. 47, 226-235 (2018).

32. Fry, A. et al. Comparison of sociodemographic and health-related characteristics of UK Biobank participants with those of the general population. Am. J. Epidemiol. 186, 1026-1034 (2017).

33. Koob, G. F. \& Volkow, N. D. Neurobiology of addiction: a neurocircuitry analysis. Lancet Psychiatry 3, 760-773 (2016).

34. Mbarek, H. et al. The genetics of alcohol dependence: twin and SNP-based heritability, and genome-wide association study based on AUDIT scores. Am. J. Med. Genet. B. Neuropsychiatr. Genet. 168, 739-748 (2015).

35. Prescott, C. A., Aggen, S. H. \& Kendler, K. S. Sex-specific genetic influences on the comorbidity of alcoholism and major depression in a populationbased sample of US twins. Arch. Gen. Psychiatry 57, 803-811 (2000).

36. Kendler, K. S., Prescott, C. A., Myers, J. \& Neale, M. C. The structure of genetic and environmental risk factors for common psychiatric and substance use disorders in men and women. Arch. Gen. Psychiatry 60 929-937 (2003).

37. Andersen, A. M. et al. Polygenic scores for major depressive disorder and risk of alcohol dependence. JAMA Psychiatry 74, 1153-1160 (2017).

38. Zhou, H. et al. Genetic risk variants associated with comorbid alcohol dependence and major depression. JAMA Psychiatry 74, 1234-1241 (2017).

39. Kreek, M. J., Nielsen, D. A., Butelman, E. R. \& LaForge, K. S. Genetic influences on impulsivity, risk taking, stress responsivity and vulnerability to drug abuse and addiction. Nat. Neurosci. 8, 1450-1457 (2005).

40. Polimanti, R. et al. The interplay between risky sexual behaviors and alcohol dependence: genome-wide association and neuroimaging support for LHPP as a risk gene. Neuropsychopharmacology 42, 598-605 (2017).

41. Grant, B. F. et al. Epidemiology of DSM-5 alcohol use disorder: results from the national epidemiologic survey on alcohol and related conditions III. JAMA Psychiatry 72, 757-766 (2015).

42. Jaffee, S., Caspi, A., Moffitt, T. E., Belsky, J. \& Silva, P. Why are children born to teen mothers at risk for adverse outcomes in young adulthood? Results from a 20-year longitudinal study. Dev. Psychopathol. 13, 377-397 (2001).

43. Martin, J., Taylor, M. J. \& Lichtenstein, P. Assessing the evidence for shared genetic risks across psychiatric disorders and traits. Psychol. Med. 48, 1759-1774 (2018)

44. Pratt, D. S. \& Kaplan, M. M. Evaluation of abnormal liver-enzyme results in asymptomatic patients. N. Engl. J. Med. 342, 1266-1271 (2000).

45. Polimanti, R. et al. Genome-wide association study of body mass index in subjects with alcohol dependence. Addict. Biol. 22, 535-549 (2017).

46. Traversy, G. \& Chaput, J.-P. Alcohol consumption and obesity: an update. Curr. Obes. Rep. 4, 122-130 (2015)
47. Schizophrenia Working Group of the Psychiatric Genomics Consortium. Biological insights from 108 schizophrenia-associated genetic loci. Nature 511, 421-427 (2014).

48. Wray, N. R. et al. Genome-wide association analyses identify 44 risk variants and refine the genetic architecture of major depression. Nat. Genet. 50, 668-681 (2018).

49. Zerbino, D. R. et al. Ensembl 2018. Nucleic Acids Res. 46(D1), D754-D761 (2018).

\section{Acknowledgements}

We thank A. Morris for providing code implementing the MANTRA Bayesian model. We thank A. Martin, A. Bloemendal, and H. Finucane for helpful conversations about the analysis protocol for admixed cohorts. We thank D. Hughes for her assistance with editorial contributions to the manuscript. We thank R. Spanagel for significant contributions as a founding member of the German Study of the Genetics of Alcoholism (GESGA). The PGC-SUD Working Group receives support from the National Institute on Drug Abuse and the National Institute of Mental Health via MH109532. We gratefully acknowledge prior support from the National Institute on Alcohol Abuse and Alcoholism. Statistical analyses for the PGC were carried out on the Genetic Cluster Computer (http://www.geneticcluster.org) hosted by SURFsara and financially supported by the Netherlands Scientific Organization (NWO 480-05-003), along with a supplement from the Dutch Brain Foundation and the VU University Amsterdam. We thank the 23 andMe research participants and employees for making this work possible. A.P. is supported by the Academy of Finland, Juselius Foundation; D.E.A. acknowledges 1K01MH093731; R.R. acknowledges AA-12502, AA-09203, AA-00145; M.K. is supported by AA009367, DA005147; M.M. is supported by AA009367, MH066140; R.A.G. is supported by AA017444; K.R. is supported by Academy of Finland; A.C.H. is supported by NIH AA07535, AA07729, AA13320, AA13321, and AA11998; A.R.D. is supported by NIH 1K01MH109765-01; A.E.A. is supported by NIH AA011408 and AA017828; A.G.W. is supported by NIH 3T32AA7464-38 S1; B.M.N. is supported by NIH U01 MH109539 and R01 MH107649; B.P.R. is supported by NIH AA011408, AA017828, and AA022537; B.S.M. is supported by NIH R01DA036525 and R01DA039408; B.T.W. is supported by NIH AA011408, AA017828, and AA022537; C. Hodgkinson. is supported by NIH and National Institute on Alcohol Abuse and Alcoholism (NIAAA) Intramural program; C.J.H. is supported by NIH DA032555, DA035804, DA011015, and DA042755; D.-S.C. is supported by NIH P20AA017830 and AA018779; W.I. is supported by NIH DA005147, DA013240, DA024417, and DA036216; S.A.B. is supported by NIH DA021905; H.d.W. is supported by NIH DA02812; M.C.S. is supported by NIH DA035804; S.V. is supported by NIH DA042755, DA037904, DA040177, and HG008983; R.P. is supported by NIH DA12690; J.G. is supported by NIH DA12690; D.B.H. is supported by NIH R01DA036583; D.G. is supported by the NIAAA Intramural program; D.M.D. is supported by NIH R01AA015416, K02AA018755, U10AA008401, and P50AA0022537; E.J.C. is supported by NIH DA023026, DA011301, and DA024413; E.O.J. is supported by NIH R01 DA044014; N.S. is supported by German Government BMBF \#01EB0130; K.M. is supported by German Government BMBF \#01EB0410; G.W.M. is supported by an NHMRC fellowship by GNT1078399; N.W. is supported by D.F.G. and B.M.B.F.; N. Dahmen is supported by D.F.G. and B.M.B.F.; J.D.B is supported by NIH R01HD060726; M.A.K. is supported by Health Research Council of New Zealand 11/792, 16/600; J.M. Boden is supported by Health Research Council of New Zealand 11/792, 16/600; J.H. is supported by Health Research Council of New Zealand 11/792, 16/600; J.F.P. is supported by Health Research Council of New Zealand 11/792, 16/600; H.H.M. is supported by NIH DA025109, DA024413, and DA016977; J.E.S. is supported by NIH K01 AA024152; J.A.K. is supported by Academy of Finland 265240, 263278, and Sigrid Juselius Foundation; J.K.H. is supported by NIH DA011015; J.L.M. is supported by NIH K01DA037914; J.M. Biernaka is supported by NIH P20AA017830 and AA25214; K.K. is supported by NIH DA011015; K.S.K. is supported by NIH P50AA0022537; L.-S.C. is supported by NIH DA038076; L.J.B. is supported by NIH R01DA036583; L.M.H. is supported by NIH AA011408 and AA017828; L.D. is supported by an Australian NHMRC Principal Research Fellowship; M.A.F. is supported by NIH/NIAAA P20AA017830; M.D.R. is supported by CSAT/SAMHSA 1H79T1026423, 1H79T1026446, AHRQ 1R18HS024208, and NIH R01DA036628; N.A.G. is supported by NIH R00DA023549; P.-H.S. is supported by NIAAA Intramural Research Program; N. Diazgranados is supported by NIAAA Intramural Research Program; M. Schwandt is supported by NIAAA Intramural Research Program; R.W. is supported by NSF GRFP DGE 1144083; P.A.F.M. is supported by NIH DA012854 and R25DA027995; J.M. is supported by Peter Boris Chair in Addictions Research; K.M.H is supported by NIH R01 HD060726, R01 HD073342, and P01 HD031921; M.B.M. is supported by NIH R01HD060726; R.K.W. is supported by NIH U01 MH094432; R.C.C. is supported by NIH R01DA036583; R.E.P. is supported by NIH K01MH113848; R.E.T. is supported by NIH R21DA043735; S.S.-R. is supported by the Frontiers of Innovation Scholars Program (FISP) and the Interdisciplinary Research Fellowship in NeuroAIDS (IRFN) and by NIH P50DA037844; S.-A.B. is supported by NIH AA011408, AA017828, AA022537, and AA022717; S.E.M. is supported by NHMRC 1103623; S.M.H. is supported by NIH R21AA024888 and K08DA032680; T.B.B. is supported by NIH MH100549; M.M.N. is supported by The BMBF-funded e:Med consortium IntegraMent 01ZX1314A, SysMedAlcoholism 01ZX1311A, and DFG-funded Excellence-Cluster ImmunoSensation; S.C. is supported by The Integrated Network IntegraMent 01ZX1314A; M. Rietschel is supported by The Integrated Network IntegraMent 01ZX1314G and SysMedAlcoholism 01ZX1311A; T.L.W. is supported by NIH R01 
DA021905 and R01 DA035804; A.M.G. is supported by NIH U10 AA08401; M.L.-P. is supported by University of Helsinki, Academy of Finland; J.L. is supported by University of Helsinki, Academy of Finland; V.M.K. is supported by NIH P20AA017830 and AA25214; W.E.C. is supported by NIH R01HD093651, R01DA036523, and P30DA023026; T.-K.C. is supported by Wellcome Trust (STRADL) 104036/Z/14/Z; M.J.A. is supported by Wellcome Trust (STRADL) 104036/Z/14/Z; A.M.M. is supported by Wellcome Trust (STRADL) 104036/Z/14/Z. A.A. is supported by NIH K02DA32573. Funding support for the Comorbidity and Trauma Study (dbGAP accession number: phs000277.v1.p1) was provided by the National Institute on Drug Abuse (R01 DA17305); GWAS genotyping services at the CIDR at The Johns Hopkins University were supported by the National Institutes of Health (contract N01-HG-65403). Funding support for the Center for Education and Drug Abuse Research (dbGAP accession number: phs001649. v1.p1) was provided by the National Institute on Drug Abuse (P50 DA005605). The Christchurch Health and Development Study (dbGAP in progress) has been supported by funding from the Health Research Council of New Zealand, the National Child Health Research Foundation (Cure Kids), the Canterbury Medical Research Foundation, the New Zealand Lottery Grants Board, the University of Otago, the Carney Centre for Pharmacogenomics, the James Hume Bequest Fund, US NIH grant MH077874, and NIDA grant "A developmental model of gene-environment interplay in SUDs" (R01DA024413) 2007-2012. COGA is supported by NIH Grant U10AA008401 from the NIAAA and the National Institute on Drug Abuse (NIDA). Funding support for this GWAS genotyping, which was performed at the Johns Hopkins University Center for Inherited Disease Research, was provided by the National Institute on Alcohol Abuse and Alcoholism, the NIH GEI (U01HG004438) and the NIH contract "High throughput genotyping for studying the genetic contributions to human disease"

(HHSN268200782096C). COGA Principal Investigators: B. Porjesz, V. Hesselbrock, H. Edenberg, L. Bierut; includes eleven different centers: University of Connecticut (V. Hesselbrock); Indiana University (H.J. Edenberg, J. Nurnberger Jr., T. Foroud); University of Iowa (S. Kuperman, J. Kramer); SUNY Downstate (B. Porjesz); Washington University in St. Louis (L. Bierut, J. Rice, K. Bucholz, A. Agrawal); University of California at San Diego (M. Schuckit); Rutgers University (J. Tischfield, A. Brooks); Department of Biomedical and Health Informatics, The Children's Hospital of Philadelphia; Department of Genetics, Perelman School of Medicine, University of Pennsylvania, Philadelphia PA (L. Almasy), Virginia Commonwealth University (D. Dick), Icahn School of Medicine at Mount Sinai (A. Goate), and Howard University (R. Taylor). Other COGA collaborators include: L. Bauer (University of Connecticut); J. McClintick, L. Wetherill, X. Xuei, Y. Liu, D. Lai, S. O'Connor, M. Plawecki, S. Lourens (Indiana University); G. Chan (University of Iowa; University of Connecticut); J. Meyers, D. Chorlian, C. Kamarajan, A. Pandey, J. Zhang (SUNY Downstate); J.-C. Wang, M. Kapoor, S. Bertelsen (Icahn School of Medicine at Mount Sinai); A. Anokhin, V. McCutcheon, S. Saccone (Washington University); J. Salvatore, F. Aliev, B. Cho (Virginia Commonwealth University); and Mark Kos (University of Texas Rio Grande Valley). A. Parsian is an NIAAA Staff Collaborator. M. Reilly was an NIAAA staff collaborator. We continue to be inspired by our memories of Henri Begleiter and Theodore Reich, founding PI and Co-PI of COGA, and also owe a debt of gratitude to other past organizers of COGA, including T. $-\mathrm{K}$. $\mathrm{Li}$, currently a consultant with COGA, as well as P. M. Conneally, R. Crowe, and W. Reich, for their critical contributions. The authors thank K. Doheny and E. Pugh from CIDR and J. Paschall from the NCBI dbGaP staff for valuable assistance with genotyping and quality control in developing the dataset available at dbGaP (phs000125.v1.p1, phs000763.v1.p1, and phs000976.v1.p1). Support for the Study of Addiction: Genetics and Environment (SAGE) was provided through the NIH Genes, Environment and Health Initiative (GEI; U01 HG004422; dbGaP study accession phs000092.v1.p1). SAGE is one of the GWAS funded as part of the Gene Environment Association Studies (GENEVA) under GEI Assistance with phenotype harmonization and genotype cleaning, as well as with general study coordination, was provided by the GENEVA Coordinating Center (U01 HG004446). Assistance with data cleaning was provided by the National Center for Biotechnology Information. Support for collection of datasets and samples was provided by COGA (U10 AA008401), the Collaborative Genetic Study of Nicotine Dependence (COGEND; P01 CA089392; see also phs000404.v1.p1), and the Family Study of Cocaine Dependence (R01 DA013423, R01 DA019963). Funding support for genotyping, which was performed at the Johns Hopkins University Center for Inherited Disease Research (CIDR), was provided by the NIH GEI (U01HG004438), the National Institute on Alcohol Abuse and Alcoholism, the National Institute on Drug Abuse, and the NIH contract "High throughput genotyping for studying the genetic contributions to human disease" (HHSN268200782096C). For GESGA, M.R. and M.M.N. were supported by the German Federal Ministry of Education and Research (BMBF) through grants BMBF 01ZX1311A (to M.R. and M.M.N.), and through grants 01ZX1314A (to M.M.N.) and 01ZX1314G (to M.R.) within the e:Med research program. The GSMS project (phs000852.v1.p1) was supported by the National Institute on Drug Abuse (U01DA024413 and R01DA11301), the National Institute of Mental Health (R01MH063970, R01MH063671, R01MH048085, K01MH093731, and K23MH080230), NARSAD, and the William T. Grant Foundation. We are grateful to all the GSMS and CCC study participants who contributed to this work. The following grants supported data collection and analysis of the Center on Antisocial Drug Dependence (CADD; dbGAP in progress): DA011015, DA012845, DA021913, DA021905, DA032555, and DA035804. Funding support for Spit for Science (dbGAP in progress) has been provided by Virginia Commonwealth University, P20 AA017828, R37AA011408, K02AA018755, and P50 AA022537 from the National Institute on Alcohol Abuse and Alcoholism, and UL1RR031990 from the National Center for Research Resources and National Institutes of Health Roadmap for Medical Research. We would like to thank the Spit for Science participants for making this study a success, as well as the many University faculty, students, and staff who contributed to the design and implementation of the project. In particular we acknowledge the contributions of the many individuals who have played a critical role data collection, generation, and cleaning, including A. Adkins, F. Aliev, E. Caraway, S. B. Cho, J. Clifford, M. Cooke, E. Do, A. Edwards, N. Goyal, L. Halberstadt, S. Hawn, R. Holloway, J. Lent, M. Lind, E. Long, J. Meyers, J. Myers, A. Moore, A. Moscati, Z. Neale, J. Opalesky, C. Overstreet, K. Pedersen, L. Rappa, B. Riley, J. Salvatore, J. Savage, C. Sun, N. Thomas, B. Webb, and J. Yan. The NIAAA data (https://btris.nih.gov/) were supported by the NIAAA Intramural Research Program (IRP). Data collection, genotyping, and analysis of the Mayo Clinic Center for Individualized Treatment of Addiction (CITA) data was supported by grants from the National Institute on Alcohol Abuse and Alcoholism (P20 AA017830, and R21 AA25214), as well as the Mayo CTSA Grant Number UL1TR000135 and SC Johnson Genomics of Addiction Program. The Mayo Clinic Biobank, which served as the source of controls for the CITA cases, was supported by Mayo Clinic Center for Individualized Medicine. Alcohol Dependence in African Americans was funded by NIH grant R01 AA017444. Brisbane Longitudinal Twin Study was supported by the United States National Institute on Drug Abuse (R00DA023549) and by the Australian Research Council (DP0343921, DP0664638, 464914, 619667, and FT110100548). Gene-Environment-Development Initiative (GEDI) - Virginia Commonwealth University (VTSABD; dbGAP in progress) was supported by the National Institute on Drug Abuse (U01DA024413 and R01DA025109), the National Institute of Mental Health (R01MH045268, R01MH055557, and R01MH068521), and the Virginia Tobacco Settlement Foundation grant 8520012. We are grateful to all the VTSABD-YAFU-TSA study participants who contributed to this work. Minnesota Center for Twin and Family Research (phs000620.v1.p1) support contributing to this publication was supported by the National Institutes of Health under award numbers DA005147, DA013240, DA024417, DA036216, AA009367, and MH066140. Funding for TwinGene is a substudy of the Swedish Twin Registry, managed by Karolinska Institutet and supported by the Swedish Research Council under grant no 2017-00641. Additional funding was provided by the Swedish Research Council (M-2005-1112), GenomEUtwin (EU/QLRT-2001-01254 and QLG2-CT-2002-01254), National Institutes of Health U01-DK 066134, the Swedish Foundation for Strategic Research (SSF), and the Heart and Lung Foundation (20070481). Substance Use Disorder Liability: Candidate System Genes study was supported by R01 DA019157 and P50 DA005605. Yale-Penn (phs000425.v1.p1 and phs000952.v1.p1) was supported by National Institutes of Health Grants RC2 DA028909, R01 DA12690, R01 DA12849, R01 DA18432, R01 AA11330, and R01 AA017535 and by the Veterans Affairs Connecticut and Philadelphia Veterans Affairs Mental Illness Research, Educational, and Clinical Centers. Australian Alcohol and Nicotine studies (phs000181.v1.p1) were supported by National Institutes of Health Grants AA07535,AA07728, AA13320, AA13321, AA14041, AA11998,

AA17688,DA012854, and DA019951; by grants from the Australian National Health and Medical Research Council (241944, 339462, 389927,389875, 389891, 389892, 389938, 442915, 442981, 496739, 552485, and 552498); by grants from the Australian Research Council(A7960034, A79906588, A79801419, DP0770096, DP0212016, and DP0343921); and by the 5th Framework Programme (FP-5) GenomEUtwin Project

(QLG2-CT-2002-01254). GWAS genotyping at Center for Inherited Disease Research was supported by a grant to the late Richard Todd, former Principal Investigator of grant AA13320. Irish Affected Sib-Pair Study of Alcohol Dependence GWAS data collection and analysis was supported by National Institute on Alcohol Abuse and Alcoholism grants P20-AA-017828 and P50-AA-022537. Sample collection was supported by R01-AA-011408. Control genotyping was supported by National Institute of Mental Health grant R01-MH-083094 and Wellcome Trust Case Control Consortium 2 grant WTCCC-084710. Netherland Twin Register (NTR) and Netherlands Study of Depression and Anxiety funding was obtained from multiple grants from the Netherlands Organization for Scientific Research (NWO) and MagW/ZonMW, including NWO-48015-001/674: Netherlands Twin Registry Repository: researching the interplay between genome and environment; Geestkracht program of the Netherlands Organization for Health Research and Development (Zon-MW 10-000-1002), Genetic determinants of risk behavior in relation to alcohol use and alcohol use disorder (ZonMWAddiction-31160008); Biobanking and Biomolecular Resources Research Infrastructure (BBMRI -NL, 184.021.007), Amsterdam Public Health research institute (APH) and Neuroscience Campus Amsterdam (NCA); and the European Science Council (ERC230374 and ERC-284167). Part of the genotyping was funded by NWO/SPI 56-46414192; the Genetic Association Information Network (GAIN) of the Foundation for the National Institutes of Health, Rutgers University Cell and DNA Repository (NIMH U24 MH068457-06), the Avera Institute for Human Genetics, Sioux Falls, South Dakota (USA), and the National Institutes of Health (NIH R01 HD042157-01A1, MH081802, and Grand Opportunity grants 1RC2 MH089951 and 1RC2 MH089995). The Finnish Twin Cohort/Nicotine Addiction Genetics-Finland study was supported by Academy of Finland (grants \# 213506 and 129680 to J.K.), NIH DA12854 (PAFM), Global Research Award for Nicotine Dependence / Pfizer Inc. (J.K.), Wellcome Trust Sanger Institute, UK and the European Community's Seventh Framework Programme ENGAGE Consortium (HEALTH-F4-2007- 201413). In Finntwin12, support for data collection and genotyping has come from National Institute of Alcohol Abuse and Alcoholism (grants AA-12502, AA-00145, and AA-09203 to R.J.R. and AA15416 and K02AA018755 to D.M.D.), the Academy of Finland (grants 100499, 205585, 118555, 141054, and 264146 to J.K.), and Wellcome Trust Sanger Institute, UK. This research uses data from Add Health, a program project directed by K. Mullan Harris and designed by J. R. Udry, P. S. Bearman, 
and K. Mullan Harris at the University of North Carolina at Chapel Hill, and funded by grant P01-HD31921 from the Eunice Kennedy Shriver National Institute of Child Health and Human Development, with cooperative funding from 23 other federal agencies and foundations. Information on how to obtain the Add Health data files is available on the Add Health website (http://www.cpc.unc.edu/addhealth). No direct support was received from grant P01-HD31921 for this analysis. The Helsinki Birth Cohort Study thanks all study participants as well as everybody involved in the Helsinki Birth Cohort Study. Helsinki Birth Cohort Study has been supported by grants from the Academy of Finland, the Finnish Diabetes Research Society, Folkhälsan Research Foundation, Novo Nordisk Foundation, Finska Läkaresällskapet, Juho Vainio Foundation, Signe and Ane Gyllenberg Foundation, University of Helsinki, Ministry of Education, Ahokas Foundation, and Emil Aaltonen Foundation. The Avon Longitudinal Study of Parents and Children (ALSPAC) is extremely grateful to all the families who took part in the ALSPAC study, the midwives for their help in recruiting them, and the whole ALSPAC team, which includes interviewers, computer and laboratory technicians, clerical workers, research scientists, volunteers, managers, receptionists, and nurses. Funding/support was from The UK Medical Research Council and Wellcome (grant ref: 102215/2/13/2) and the University of Bristol provide core support for ALSPAC. This publication is the work of the authors and A.C.E. will serve as guarantor for the contents of this paper. A comprehensive list of grants funding is available on the ALSPAC website (http://www.bristol.ac.uk/alspac/ external/documents/grant-acknowledgements.pdf). This research was specifically funded by NIH grants AA021399. GWAS data were generated by Sample Logistics and Genotyping Facilities at Wellcome Sanger Institute and LabCorp (Laboratory Corporation of America) using support from 23andMe. Generation Scotland is gratefu to the families who took part in GS, the GPs and Scottish School of Primary Care for their help in recruiting them, and the whole GS team that includes academic researchers, clinic staff, laboratory technicians, clerical workers, IT staff, statisticians, and research managers. Generation Scotland received core support from the Chief Scientist Office of the Scottish Government Health Directorates (CZD/16/6) and the Scottish Funding Council (HR03006). Genotyping of the GS samples was carried out by the Genetics Core Laboratory at the Wellcome Trust Clinical Research Facility, Edinburgh, Scotland, and was funded by the Medical Research Council UK and the Wellcome Trust (Wellcome Trust Strategic Award 'STratifying Resilience and Depression Longitudinally' (STRADL) Reference 104036/Z/14/Z). LD Hub (http://ldsc.broadinstitute.org/) is grateful to the following GWAS studies, databases and consortia who have kindly made their summary data available: ADIPOGen (Adiponectin genetics consortium), C4D (Coronary Artery Disease Genetics Consortium), CARDIoGRAM (Coronary ARtery DIsease Genome wide Replication and Meta-analysis), CKDGen (Chronic Kidney Disease Genetics consortium), dbGAP (database of Genotypes and Phenotypes), DIAGRAM (DIAbetes Genetics Replication And Meta-analysis), ENIGMA (Enhancing Neuro Imaging Genetics through Meta Analysis), EAGLE (EArly Genetics \& Lifecourse Epidemiology Consortium, excluding 23andMe), EGG (Early Growth Genetics Consortium), GABRIEL (A Multidisciplinary Study to Identify the Genetic and Environmental Causes of Asthma in the European Community), GCAN (Genetic Consortium for Anorexia Nervosa), GEFOS (GEnetic Factors for OSteoporosis Consortium), GIANT (Genetic Investigation of ANthropometric Traits), GIS (Genetics of Iron Status consortium), GLGC (Global Lipids Genetics Consortium), GPC (Genetics of Personality Consortium), GUGC (Global Urate and Gout consortium), HaemGen (hematological and platelet traits genetics consortium), HRgene (Heart Rate consortium), IIBDGC (International Inflammatory Bowel Disease Genetics Consortium), ILCCO (International Lung Cancer Consortium), IMSGC (International Multiple Sclerosis Genetic Consortium), MAGIC (Meta-Analyses of Glucose and Insulin-related traits Consortium), MESA (Multi-Ethnic Study of Atherosclerosis), PGC (Psychiatric Genomics Consortium), Project MinE consortium, ReproGen (Reproductive Genetics Consortium), SSGAC (Social Science Genetics Association Consortium), TAG (Tobacco and Genetics Consortium), TRICL (Transdisciplinary Research in Cancer of the Lung consortium), and UK Biobank. We additionally thank the groups who directly shared GWAS results. We would like to acknowledge all participating groups of the International Cannabis Consortium, and in particular the members of the working group including S. Stringer, C. Minica, K. Verweij, H. Mbarek, E. Derks, N. Gillespie, and J. Vink. Thanks also to the ENIGMA consortium for providing GWAS results on subcortical brain volumes (available from http://enigma.ini.usc.edu/research/download-enigma-gwas-results/). Finally, we acknowledge the valuable contribution of groups who have publicly released summary statistics from their respective GWAS. Specifically, thanks to researchers from Schumann et al.${ }^{16}$ including the CHARGE+ and AlcGen consortia (results available at https://grasp.nhlbi.nih.gov/FullResults.aspx) and to all members of Psychiatric Genomics Consortium (PGC; results available for download at http://www.med.unc.edu/pgc/ results-and-downloads), in particular the working groups on attention deficit/ hyperactivity disorder (ADHD; chaired by S. Faraone), autism spectrum disorder (ASD; chaired by M. Daly and B. Devlin), and eating disorders (ED; chaired by C. Bulik and G. Breen). Similar thanks to all of the participating groups in the Lundbeck Foundation Initiative for Integrative Psychiatric Research (iPSYCH) consortium for their participation in the ADHD and ASD meta-analyses.

\section{Author contributions}

R.K.W., H.J.E., J.G., and A.A. conceived the analyses, wrote the first draft and prepared all drafts for submission; R.K.W. conducted primary analyses; R.P., E.C.J., and J.N.M. conducted additional analyses. H.J.E., J.G., A.A., and B.N. supervised all analyses. M.J.A., A.E.A., F.A., S.-A.B., A.B., S.B., J.M. Biernaka, T.B.B., L.-S.C., T.-K.C., Y.-L.C., F.D.,
A.R.D., A.C.E., P.F., J.C.F., L.F., J.F., I.G., S.G., L.M.H., A.M.H., S.M.H., S.H.-H., S.H., C. Hodgkinson, P.H. J.J.H., M.A.K., M.A.-K., B.K., J.L., M.L.-P., D.L., L.L., A.L., B.S.M., H.M., A.M.M., M.B.M., J.L.M., Y.M., T.P., J.F.P., R.E.P., S.R., E.R., N.L.S., J.E.S., S.S.-R., M. Schwandt, R.S., F.S., J. Strohmaier, N.T., J.-C.W., B.T.W., R.W., L.W. and A.G.W. prepared individual datasets and, in some cases, conducted analyses and provided summary statistics or results. The 23 andMe Research Team, J.D.B., D.C., D.-S.C., W.E.C., R.C.C. N. Dahmen, L.D., B.W.D., S.L.E., M.A.F., W.G., C. Hayward, M.I., M.K., F.K., J.K., S.K., S.L., M.T.L., W.M., K.M., S.M., B.M.-M., A.D.M., J.I.N., A.P., U.P., K.R., M.D.R., M. Ridinger, N.S., M.A.S., M. Soyka, J.T., S.W., N.W., and P.Z. provided critical support regarding phenotypes and data in individual datasets; D.E.A., J.M. Boden, D.I.B., L.J.B., S.A.B., K.K.B., S.C., E.J.C., H.d.W., N. Diazgranados, D.M.D., J.G.E., L.A.F., T.M.F, N.A.G., A.M.G., D.G., R.A.G., D.B.H., K.M.H., A.C.H., V.H., J.K.H., C.J.H., J.H., W.I., E.O.J., J.A.K., V.M.K., K.S.K., H.R.K., K.K., P.L., P.A.L., M.M., J.M., P.A.F.M., H.H.M., P.M., N.G.M., S.E.M., G.W.M., E.C.N., M.M.N., A.A.P., N.L.P., B.W.J.H.P., B.P., J.P.R., M. Rietschel, B.P.R., R.R., D.R., P.-H.S., J. Silberg, M.C.S., R.E.T., M.M.V., S.V., T.L.W., J.B.W., and H.Z., as well as H.J.E. and J.G., facilitated data collection and provided critical phenotypic and analytic feedback for individual studies. B.M.N. and A.A. provided resource support. All authors reviewed the manuscript and approved it for submission.

\section{Competing interests}

L.J.B., A.M.G., J.P.R., J.-C.W. and the spouse of N.L.S. are listed as inventors on Issued US Patent 8080,371, "Markers for Addiction" covering the use of certain SNPs in determining the diagnosis, prognosis, and treatment of addiction. N.W. has received funding from the German Research Foundation (DFG) and Federal Ministry of Education and Research Germany (BMBF); he has received speaker's honoraria and travel funds from Janssen-Cilag and Indivior. He took part in industry-sponsored multicenter randomized trials by D\&A Pharma and Lundbeck. M. Ridinger received compensation from Lundbeck Switzerland and Lundbeck institute for advisory boards and expert meetings, and from Lundbeck and Lilly Suisse for workshops and presentations. K.M. received honoraria from Lundbeck, Pfizer, Novartis, and AbbVie. K.M. also received Honoraria (Advisory Board) from Lundbeck and Pfizer and speaker fees from Janssen Cilag. H.K. has been an advisory board member, consultant, or continuing medical education speaker for Indivior, Lundbeck, and Otsuka. He is a member of the American Society of Clinical Psychopharmacology's Alcohol Clinical Trials Initiative, which was sponsored in the past three years by AbbVie, Alkermes, Amygdala Neurosciences, Arbor Pharmaceuticals, Ethypharm, Indivior, Lilly, Lundbeck, Otsuka, and Pfizer. H.K. and J.G. are named as inventors on PCT patent application \#15/878,640, entitled "Genotype-guided dosing of opioid agonists," filed 24 January 2018. P.F., S.L.E., and members of the 23 andMe Research Team are employees of 23 andMe. M.A.F. has received grant support from Assurex Health, Mayo Foundation, Myriad, NIAAA, National Institute of Mental Health (NIMH), and Pfizer; he has been a consultant for Intra-Cellular Therapies, Inc., Janssen, Mitsubishi Tanabe Pharma Corporation, Myriad, Neuralstem Inc., Otsuka American Pharmaceutical, Sunovion, and Teva Pharmaceuticals. H.d.W. has received support from Insys Therapeutics and Indivior for studies unrelated to this project, and she has consulted for Marinus and Jazz Pharmaceuticals, also unrelated to this project. T.L.W. has previously received funds from ABMRF. J.N. is an investigator for Janssen and Assurex. M.M.N. has received honoraria from the Lundbeck Foundation and the Robert Bosch Stiftung for membership on advisory boards. M. Ridinger has received honoraria from Lundbeck Switzerland and the Lundbeck Institute for membership of advisory boards and participation in expert meetings, and from Lundbeck and Lilly Suisse for workshops and presentations. N.S. has received honoraria from Abbvie, Sanofi-Aventis, Reckitt Benckiser, Indivior, Lundbeck, and Janssen-Cilag for advisory board membership and the preparation of lectures, manuscripts, and educational materials. Since 2013, N.S. has also participated in clinical trials financed by Reckitt Benckiser and Indivior. N.W. received speaker's honoraria and travel expenses from Janssen-Cilag and Indivior; has also participated in industry-sponsored multicenter randomized trials conducted by D\&A Pharma and Lundbeck. W.G. has received symposia support from Janssen-Cilag GmbH, Neuss, Lilly Deutschland GmbH, Bad Homburg, and Servier, Munich, and is a member of the Faculty of the Lundbeck International Neuroscience Foundation (LINF), Denmark. J.A.K. has provided consultations on nicotine dependence for Pfizer (Finland) 2012-2015. In the past three years, L.D. has received investigator-initiated untied educational grants for studies of opioid medications in Australia from Indivior, Mundipharma, and Seqirus. B.M.N. is a member of the scientific advisory board for Deep Genomics and has consulted for Camp4 Therapeutics Corporation, Merck \& Co., and Avanir Pharmaceuticals, Inc. A.A. previously received peer-reviewed funding and travel reimbursement from ABMRF for unrelated research.

\section{Additional information}

Supplementary information is available for this paper at https://doi.org/10.1038/ s41593-018-0275-1.

Reprints and permissions information is available at www.nature.com/reprints. Correspondence and requests for materials should be addressed to J.G. or H.J.E. or A.A Publisher's note: Springer Nature remains neutral with regard to jurisdictional claims in published maps and institutional affiliations.

(c) The Author(s), under exclusive licence to Springer Nature America, Inc. 2018 
Raymond K. Walters $\mathbb{1}^{1,2}$, Renato Polimanti ${ }^{3}$, Emma C. Johnson ${ }^{4}$, Jeanette N. McClintick ${ }^{5}$, Mark J. Adams ${ }^{6}{ }^{6}$, Amy E. Adkins ${ }^{7}$, Fazil Aliev ${ }^{8}$, Silviu-Alin Bacanu ${ }^{9}$, Anthony Batzler ${ }^{10}$, Sarah Bertelsen"1, Joanna M. Biernacka ${ }^{12}$, Tim B. Bigdeli ${ }^{13}$, Li-Shiun Chen ${ }^{4}$, Toni-Kim Clarke ${ }^{6}$, Yi-Ling Chou ${ }^{4}$, Franziska Degenhardt ${ }^{14}$, Anna R. Docherty ${ }^{15}$, Alexis C. Edwards ${ }^{16}$, Pierre Fontanillas ${ }^{17}$, Jerome C. Foo ${ }^{18}$, Louis Fox ${ }^{4}$, Josef Frank ${ }^{18}$, Ina Giegling ${ }^{19}$, Scott Gordon ${ }^{20}$, Laura M. Hack ${ }^{21}$, Annette M. Hartmann ${ }^{19}$, Sarah M. Hartz ${ }^{4}$, Stefanie Heilmann-Heimbach ${ }^{14}$, Stefan Herms ${ }^{14,22}$, Colin Hodgkinson ${ }^{23}$, Per Hoffmann ${ }^{14,22}$, Jouke Jan Hottenga ${ }^{24}$, Martin A. Kennedy ${ }^{25}$, Mervi Alanne-Kinnunen ${ }^{26}$, Bettina Konte ${ }^{19}{ }^{19}$, Jari Lahti ${ }^{27,28}$, Marius Lahti-Pulkkinen ${ }^{28}{ }^{28}$, Dongbing Lai ${ }^{29}$, Lannie Ligthart ${ }^{24}$, Anu Loukola26, Brion S. Maher ${ }^{30}$, Hamdi Mbarek ${ }^{24}$, Andrew M. Mclntosh ${ }^{31}$, Matthew B. McQueen ${ }^{32}$, Jacquelyn L. Meyers ${ }^{33}$, Yuri Milaneschii ${ }^{34}$, Teemu Palviainen ${ }^{26}$, John F.Pearson ${ }^{35}$, Roseann E. Peterson ${ }^{16}$, Samuli Ripatti1,2,26,36, Euijung Ryu ${ }^{37}$, Nancy L. Saccone ${ }^{38}$, Jessica E. Salvatore ${ }^{8,16}$, Sandra Sanchez-Roige ${ }^{39}$, Melanie Schwandt ${ }^{40}$, Richard Sherva ${ }^{41}$, Fabian Streit ${ }^{18}{ }^{18}$, Jana Strohmaier ${ }^{18}$, Nathaniel Thomas ${ }^{7}$, Jen-Chyong Wang ${ }^{11}$, Bradley T. Webb ${ }^{9}$, Robbee Wedow 1,2,42,43, Leah Wetherill29, Amanda G. Wills ${ }^{44}$, 23andMe Research Team ${ }^{45}$, Jason D. Boardman ${ }^{46}$, Danfeng Chen ${ }^{2}$, Doo-Sup Choi ${ }^{47}$, William E. Copeland ${ }^{48}$, Robert C. Culverhouse ${ }^{49}$, Norbert Dahmen ${ }^{50}$, Louisa Degenhardt ${ }^{51}$, Benjamin W. Domingue ${ }^{52}$, Sarah L. Elson ${ }^{17}$, Mark A. Frye ${ }^{53}$, Wolfgang Gäbel ${ }^{54}$, Caroline Hayward (1) ${ }^{55}$, Marcus Ising ${ }^{56}$, Margaret Keyes ${ }^{57}$, Falk Kiefer ${ }^{58}$, John Kramer ${ }^{59}$, Samuel Kuperman ${ }^{59}$, Susanne Lucae ${ }^{56}$, Michael T. Lynskey ${ }^{60}$, Wolfgang Maier ${ }^{61}$, KarlMann ${ }^{58}$, Satu Männistö ${ }^{62}$, Bertram Müller-Myhsok ${ }^{63}$, Alison D. Murray ${ }^{64}$, John I. Nurnberger ${ }^{29,65}$, Aarno Palotie ${ }^{1,2,26,66}$, Ulrich Preuss ${ }^{19,67}$, Katri Räikkönen ${ }^{28}$, Maureen D Reynolds ${ }^{68}$, Monika Ridinger ${ }^{69}$, Norbert Scherbaum ${ }^{70}$, Marc A. Schuckitt ${ }^{39}$, MichaelSoyka ${ }^{71,72}$, Jens Treutlein ${ }^{18}$, Stephanie Witt ${ }^{18}$, Norbert Wodarz ${ }^{73}$, Peter Zill72, Daniel E. Adkins ${ }^{15,74}$, Joseph M. Boden ${ }^{25}$, Dorret I. Boomsma ${ }^{24}$, Laura J. Bierut ${ }^{4}$, Sandra A. Brown ${ }^{39,75}$, Kathleen K. Bucholz ${ }^{4}$, Sven Cichon ${ }^{22}$, E. Jane Costello ${ }^{48}$, Harriet de Wit ${ }^{76}$, Nancy Diazgranados ${ }^{76}$, Danielle M. Dick ${ }^{7,77}$, Johan G. Eriksson ${ }^{78}$, Lindsay A. Farrer ${ }^{41,79}$, Tatiana M. Foroud ${ }^{29}$, Nathan A. Gillespie ${ }^{16}$, Alison M. Goate ${ }^{11}{ }^{11}$, David Goldman ${ }^{23,40}$, Richard A. Grucza ${ }^{4}$, Dana B. Hancock ${ }^{80}$, Kathleen Mullan Harris ${ }^{81}$, Andrew C. Heath ${ }^{4}$, Victor Hesselbrock ${ }^{82}$, John K. Hewitt ${ }^{83}$, Christian J. Hopfer ${ }^{84}$, John Horwood25, William lacono57, Eric O. Johnson ${ }^{85}$, Jaakko A. Kaprio ${ }^{26,36}$, Victor M. Karpyak ${ }^{53}$, Kenneth S. Kendler ${ }^{9}$, Henry R. Kranzler ${ }^{86}{ }^{8}$, Kenneth Krauter ${ }^{87}$, Paul Lichtenstein ${ }^{88}$, Penelope A. Lind (1020, Matt McGue ${ }^{57}$, James MacKillop ${ }^{89}$, Pamela A. F. Madden ${ }^{4}$, Hermine H. Maes ${ }^{90}$, Patrik Magnusson ${ }^{88}$, Nicholas G. Martin ${ }^{20}$, Sarah E. Medland ${ }^{20}$, Grant W. Montgomery ${ }^{(1)}$, Elliot C. Nelson ${ }^{4}$, Markus M. Nöthen ${ }^{92}$, Abraham A. Palmer ${ }^{39,93}$, Nancy L. Pedersen ${ }^{88}$, Brenda W. J. H. Penninx ${ }^{34}$, Bernice Porjesz ${ }^{33}$, John P. Rice ${ }^{4}$, Marcella Rietschel ${ }^{18}$, Brien P. Riley ${ }^{9}$, Richard Rose ${ }^{94}$, Dan Rujescu ${ }^{19}$, Pei-Hong Shen ${ }^{23}$, Judy Silberg ${ }^{16}$, Michael C. Stallings ${ }^{83}$, Ralph E. Tarter ${ }^{68}$, Michael M. Vanyukov ${ }^{68}$, Scott Vrieze ${ }^{57}$, Tamara L. Wall ${ }^{39}$, John B. Whitfield (1) ${ }^{20}$, Hongyu Zhao ${ }^{95}$, Benjamin M. Neale $\mathbb{( D )}^{1,2}$, Joel Gelernter ${ }^{96 \star}{ }^{96}$ Howard J. Edenberg ${ }^{(15,29 \star}$ and Arpana Agrawal ${ }^{4 \star}{ }^{4 \star}$ 
'Analytic and Translational Genetics Unit, Department of Medicine, Massachusetts General Hospital and Harvard Medical School, Boston, MA, USA. ${ }^{2}$ Stanley Center for Psychiatric Research, Broad Institute of MIT and Harvard, Cambridge, MA, USA. ${ }^{3}$ Department of Psychiatry, Yale School of Medicine and Veterans Affairs Connecticut Healthcare Center, West Haven, CT, USA. ${ }^{4}$ Washington University School of Medicine, Department of Psychiatry, St. Louis, MO, USA. ${ }^{5}$ Department of Biochemistry and Molecular Biology, Indiana University School of Medicine, Indianapolis, IN, USA. ${ }^{6}$ University of Edinburgh, Division of Psychiatry, Edinburgh, UK. 'Department of Psychology \& College Behavioral and Emotional Health Institute, Virginia Commonwealth University, Richmond, VA, USA. ${ }^{8}$ Virginia Commonwealth University, Department of Psychology, Richmond, VA, USA. ${ }^{9}$ Virginia Commonwealth University Alcohol Research Center; Virginia Institute for Psychiatric and Behavioral Genetics; Department of Psychiatry, Virginia Commonwealth University, Richmond, VA, USA. ${ }^{10}$ Mayo Clinic, Psychiatric Genomics and Pharmacogenomics Program, Rochester, MN, USA. "Icahn School of Medicine at Mount Sinai, Department of Neuroscience, New York, NY, USA. ${ }^{12}$ Mayo Clinic, Department of Health Sciences Research, and Department of Psychiatry and Psychology, Rochester, MN, USA. ${ }^{33}$ Department of Psychiatry and Behavioral Sciences, State University of New York Downstate Medical Center, Brooklyn, NY, USA. ${ }^{14}$ Institute of Human Genetics, University of Bonn; and Department of Genomics, Life \& Brain Center, University of Bonn, Bonn, Germany. ${ }^{15}$ University of Utah, Department of Psychiatry, Salt Lake City, UT, USA. ${ }^{16}$ Virginia Commonwealth University, Virginia Institute for Psychiatric and Behavioral Genetics, Department of Psychiatry, Richmond, VA, USA. ${ }^{1723 a n d M e, ~ I n c, ~ M o u n t a i n ~ V i e w, ~ C A, ~ U S A . ~}{ }^{18}$ Department of Genetic Epidemiology in Psychiatry, Central Institute of Mental Health, Medical Faculty Mannheim, Heidelberg University, Mannheim, Germany. ${ }^{19}$ Martin-Luther-University Halle-Wittenberg, Department of Psychiatry, Psychotherapy and Psychosomatics, Halle, Germany. ${ }^{20}$ QIMR Berghofer Medical Research Institute, Brisbane, Queensland, Australia. ${ }^{21}$ Department of Psychiatry and Behavioral Sciences, Emory University School of Medicine, Atlanta, GA, USA. ${ }^{22}$ Human Genomics Research Group, Department of Biomedicine, University of Basel Institute of Medical Genetics and Pathology, University Hospital Basel, Basel, Switzerland. ${ }^{23} \mathrm{NIH} / \mathrm{NIAAA}$, Laboratory of Neurogenetics, Bethesda, MD, USA. ${ }^{24}$ Department of Biological Psychology, Amsterdam Public Health Research Institute, Vrije Universiteit Amsterdam, Amsterdam, The Netherlands. ${ }^{25}$ University of Otago, Christchurch, New Zealand. ${ }^{26}$ Institute for Molecular Medicine Finland (FIMM), University of Helsinki, Helsinki, Finland. ${ }^{27} \mathrm{Helsinki}$ Collegium for Advanced Studies, University of Helsinki, Helsinki, Finland. ${ }^{28}$ Department of Psychology and Logopedics, University of Helsinki, Helsinki, Finland. ${ }^{29}$ Department of Medical and Molecular Genetics, Indiana University School of Medicine, Indianapolis, IN, USA. ${ }^{30}$ Johns Hopkins Bloomberg School of Public Health, Baltimore, MD, USA. ${ }^{31}$ University of Edinburgh, Division of Psychiatry, Centre for Cognitive Ageing and Cognitive Epidemiology, Edinburgh, UK. ${ }^{32}$ Department of Integrative Physiology, University of Colorado Boulder, Boulder, CO, USA. ${ }^{33}$ Henri Begleiter Neurodynamics Laboratory, Department of Psychiatry and Behavioral Sciences, SUNY Downstate Medical Center, Brooklyn, NY, USA. ${ }^{34}$ Department of Psychiatry, Amsterdam Public Health Research Institute, VU University Medical Center/GGz inGeest, Amsterdam, The Netherlands. ${ }^{35}$ Biostatistics and Computational Biology Unit, University of Otago, Christchurch, New Zealand. ${ }^{36}$ Department of Public Health, University of Helsinki, Helsinki, Finland. ${ }^{37}$ Mayo Clinic, Department of Health Sciences Research, Rochester, MN, USA. ${ }^{38}$ Washington University School of Medicine, Department of Genetics, St. Louis, MO, USA. ${ }^{39}$ University of California San Diego, Department of Psychiatry, San Diego, CA, USA. ${ }^{40}$ NIH/NIAAA, Office of the Clinical Director, Bethesda, MD, USA. ${ }^{41}$ Department of Medicine (Biomedical Genetics), Boston University School of Medicine, Boston, MA, USA. ${ }^{42}$ Department of Epidemiology, Harvard T.H. Chan School of Public Health, Boston, MA, USA. ${ }^{43}$ Department of Sociology, Harvard University, Cambridge, MA, USA. ${ }^{44}$ University of Colorado School of Medicine, Department of Pharmacology, Aurora, CO, USA. ${ }^{45} \mathrm{~A}$ list of members and affiliations appears at the end of the paper. ${ }^{46}$ Institute of Behavioral Science and Department of Sociology, University of Colorado, Boulder, CO, USA. ${ }^{47}$ Mayo Clinic, Department of Molecular Pharmacology and Experimental Therapeutics, Rochester, MN, USA. ${ }^{48}$ Duke University Medical Center, Department of Psychiatry and Behavioral Sciences, Durham, NC, USA. ${ }^{49}$ Washington University School of Medicine, Department of Medicine and Division of Biostatistics, St. Louis, MO, USA. ${ }^{50}$ Department of Psychiatry, University of Mainz, Mainz, Germany. ${ }^{51}$ National Drug and Alcohol Research Centre, University of New South Wales, Sydney, New South Wales, Australia. ${ }^{52}$ Stanford University Graduate School of Education, Stanford, CA, USA. ${ }^{53}$ Mayo Clinic, Department of Psychiatry and Psychology, Rochester, MN, USA. ${ }^{54}$ Department of Psychiatry and Psychotherapy, University of Düsseldorf, Düsseldorf, Germany. ${ }^{55}$ MRC Human Genetics Unit, Institute of Genetics and Molecular Medicine, University of Edinburgh, Edinburgh, UK. ${ }^{56}$ Max-Planck-Institute of Psychiatry, Munich, Germany. ${ }^{57}$ University of Minnesota, Department of Psychology, Minneapolis, MN, USA. ${ }^{58}$ Department of Addictive Behavior and Addiction Medicine, Central Institute of Mental Health, Medical Faculty Mannheim, Heidelberg University, Heidelberg, Germany. ${ }^{59}$ University of lowa Roy J and Lucille A Carver College of Medicine, Department of Psychiatry, lowa City, IA, USA. ${ }^{60}$ Addictions Department, Institute of Psychiatry, Psychology \& Neuroscience, King's College London, London, UK. ${ }^{61}$ Department of Psychiatry, University of Bonn, Bonn, Germany. ${ }^{62}$ Institute for Health and Welfare, Helsinki, Finland. ${ }^{63}$ Department of Statistical Genetics, Max-Planck-Institute of Psychiatry, Munich, Germany. ${ }^{64}$ The Institute of Medical Sciences, Aberdeen Biomedical Imaging Centre, University of Aberdeen, Aberdeen, UK. ${ }^{65}$ Department of Psychiatry, Indiana University School of Medicine, Indianapolis, IN, USA. ${ }^{66}$ Department of Medicine, Department of Neurology and Department of Psychiatry, Massachusetts General Hospital, Boston, MA, USA. ${ }^{67}$ Vitos Hospital Herborn, Department of Psychiatry and Psychotherapy, Herborn, Germany. ${ }^{68}$ University of Pittsburgh, School of Pharmacy, Pittsburgh, PA, USA. ${ }^{69}$ Department of Psychiatry and Psychotherapy, University of Regensburg Psychiatric Health Care Aargau, Regensburg, Germany. ${ }^{70}$ LVR-Hospital Essen, Department of Psychiatry and Psychotherapy, Department of Addictive Behaviour and Addiction Medicine, Medical Faculty, University of Duisburg-Essen, Duisburg, Germany. ${ }^{71}$ Medical Park Chiemseeblick in Bernau-Felden, Chiemsee, Germany. ${ }^{72}$ Psychiatric Hospital, Ludwig-Maximilians-University, Munich, Germany. ${ }^{73}$ Department of Psychiatry and Psychotherapy, University of Regensburg, Regensburg, Germany. ${ }^{74}$ University of Utah, Department of Sociology, Salt Lake City, UT, USA. ${ }^{75}$ University of California, San Diego School of Medicine, Department of Psychology, San Diego, CA, USA. ${ }^{76}$ NIAAA Intramural Research Program, Bethesda, MD, USA. ${ }^{77}$ Department of Human \& Molecular Genetics, Virginia Commonwealth University, Richmond, VA, USA. ${ }^{78}$ Department of General Practice and Primary Health Care, University of Helsinki, and National Institute for Health and Welfare, Helsinki, Finland. ${ }^{79}$ Departments of Neurology, Ophthalmology, Epidemiology, and Biostatistics, Boston University Schools of Medicine and Public Health, Boston, MA, USA. ${ }^{80}$ Center for Omics Discovery and Epidemiology, Behavioral Health Research Division, RTI International, Research Triangle Park, NC, USA. ${ }^{81}$ Department of Sociology and Carolina Population Center, University of North Carolina at Chapel Hill, Chapel Hill, NC, USA. ${ }^{2}$ University of Connecticut School of Medicine, Department of Psychiatry, Farmington, CT, USA. ${ }^{83}$ University of Colorado Boulder, Institute for Behavioral Genetics, Boulder, CO, USA. ${ }^{84}$ University of Colorado Denver, School of Medicine, Denver, CO, USA. ${ }^{55}$ RTI International, Fellows Program, Research Triangle Park, NC, USA. ${ }^{86}$ University of Pennsylvania Perelman School of Medicine, Center for Studies of Addiction, Department of Psychiatry and VISN 4 MIRECC, Crescenz VAMC, Philadelphia, PA, USA. ${ }^{87}$ University of Colorado Boulder, Department of Molecular, Cellular, and Developmental Biology, Boulder, CO, USA. ${ }^{88}$ Department of Medical Epidemiology and Biostatistics, Karolinska Instituet, Stockholm, Sweden. ${ }^{89}$ Peter Boris Centre for Addictions Research, McMaster University/St. Joseph's Healthcare Hamilton; Michael G. DeGroote Centre for Medicinal Cannabis Research, Hamilton, Ontario, Canada. ${ }^{90}$ Virginia Commonwealth University, Virginia Institute for Psychiatric and Behavioral Genetics, Richmond, VA, USA. ${ }^{11}$ The Institute for Molecular Bioscience, University of Queensland, Brisbane, Queensland, Australia. ${ }^{92}$ Institute of Human Genetics, University of Bonn School of Medicine \& University Hospital Bonn, Bonn, Germany. ${ }^{93}$ University of California San Diego, Institute for Genomic Medicine, San Diego, CA, USA. ${ }^{94}$ Department of Psychological \& Brain Sciences, Indiana University Bloomington, Bloomington, IN, USA. ${ }^{95}$ Department of Biostatistics, Yale School of Public Health, Yale University, New Haven, CT, USA. ${ }^{96}$ Departments of Psychiatry, Genetics, and Neuroscience, Yale University School of Medicine, Veterans Affairs Connecticut Healthcare System, New Haven, CT, USA. *e-mail: joel.gelernter@yale.edu; edenberg@iu.edu; Arpana@wustl.edu 


\section{3andMe Research Team}

Michelle Agee ${ }^{17}$, Babak Alipanahi ${ }^{17}$, Adam Auton ${ }^{17}$, Robert K. Bell ${ }^{17}$, Katarzyna Bryc ${ }^{17}$, Sarah L. Elson ${ }^{17}$, Pierre Fontanillas ${ }^{17}$, Nicholas A. Furlotte ${ }^{17}$, David A. Hinds ${ }^{17}$, Karen E. Huber ${ }^{17}$, Aaron Kleinman ${ }^{17}$, Nadia K. Litterman ${ }^{17}$, Jennifer C. McCreight ${ }^{17}$, Matthew H. Mclntyre ${ }^{17}$, Joanna L. Mountain ${ }^{17}$, Elizabeth S. Noblin ${ }^{17}$, Carrie A. M. Northover ${ }^{17}$, Steven J. Pitts ${ }^{17}$, J. Fah Sathirapongsasuti ${ }^{17}$, Olga V. Sazonova"7, Janie F. Shelton ${ }^{17}$, Suyash Shringarpure ${ }^{17}$, Chao Tian ${ }^{17}$, Joyce Y. Tung ${ }^{17}$, Vladimir Vacic ${ }^{17}$ and Catherine H. Wilson ${ }^{17}$ 


\section{Methods}

Samples. The Substance Use Disorders working group of the Psychiatric Genomics Consortium (PGC-SUD ${ }^{14}$ ) collected individual genotypic data from 14 case-control studies and nine family-based studies and summary statistics from GWAS of AD from five additional cohorts (Table 1). AD was defined as meeting criteria for a DSM-IV ${ }^{2}$ (or, for one cohort, DSM-IIIR ${ }^{50}$; a very similar construct; see Supplementary Note) diagnosis of AD. Diagnoses were derived either from clinician ratings or semistructured interviews. Excepting three cohorts with population-based controls $(n=7,015)$, all controls were screened for AD. Individuals with no history of drinking alcohol and those meeting criteria for DSM-IV alcohol abuse were excluded as controls where possible (Supplementary Information and see Nature Research Reporting Summary). This study was approved by the institutional review board (IRB) of Washington University in St. Louis and was conducted in accordance with all relevant ethical regulations. Each contributing cohort obtained informed consent from their participants and received ethics approvals for their study protocols from their respective review boards in accordance with applicable regulations.

Quality control and imputation. Data for the cohorts that shared raw genotypes were deposited to a secure server for uniform quality control (QC). QC and imputation of the 14 case-control studies was performed using the Ricopili pipeline (https://github.com/Nealelab/ricopili). For the nine family-based cohorts, an equivalent pipeline, Picopili (https://github.com/Nealelab/picopili), was developed for QC, imputation, and analysis appropriate for diverse family structures, including twins, sibships, and extended pedigrees (Supplementary Information).

After initial sample and variant QC, principal components analysis (PCA) was used to identify population outliers for exclusion and to stratify samples in each study by continental ancestry. Identified EU and AA ancestry populations were confirmed by PCA using the 1000 Genomes Project reference panel ${ }^{21}$ (Supplementary Fig. 12). Ancestry within these two groups was accounted for with principal components. Final sample and variant QC, including filters for call rate, heterozygosity, and departure from Hardy-Weinberg equilibrium (HWE), was then performed within each ancestry group in each cohort. Samples were also filtered for cryptic relatedness and for departures from reported pedigree structures (Supplementary Information and Nature Research Reporting Summary).

Each cohort was imputed using SHAPEIT ${ }^{51}$ and IMPUTE2 ${ }^{52}$, using the cosmopolitan (all ancestries) 1000 Genomes Project reference panel consistent with prior recommendations $\mathrm{s}^{53}$ (see also refs ${ }^{47,54,55}$ ). Concordance of MAFs with the reference panel was verified before imputation, with SNPs in EU cohorts compared to MAF in European population samples and AA cohorts compared to MAF in African population samples (Supplementary Information). Instances of cryptic relatedness between cohorts were identified and excluded after imputation (Supplementary Information). Imputed SNPs were then filtered for INFO score $>0.8$ and allele frequency $>0.01$ before analysis.

Association analysis. A GWAS of AD status was performed within each ancestry stratum of each sample using an association model appropriate for the study design (Table 1 and Supplementary Table 1). For case-control studies, GWAS was performed using logistic regression with imputed dosages. For family-based studies of small, simple pedigrees (for example, sibships), association with imputed genotypes was tested using generalized estimating equations (GEE). For more complex pedigrees, imputed genotypes were tested using logistic mixed models. Sex was included as a covariate, along with principal components to control for population structure (Supplementary Information, Supplementary Note, and Supplementary Figs. 13 and 14).

In addition to this primary analysis, subsets of genetically unrelated individuals were selected from each family-based cohort (i.e., the most severe case in each family, by symptom count, was selected, followed by selection of unrelated/married-in controls) and used to perform a conventional case-control GWAS using logistic regression. This was used in place of the family-based GWAS for estimation of effect sizes and LD score regression analyses (Supplementary Table 2).

Genome-wide meta-analysis. The primary discovery meta-analysis of all ancestry stratified GWAS ( $\left.n_{\text {case }}=14,904 ; n_{\text {control }}=37,944\right)$ was conducted in METAL ${ }^{56}$. As the different study designs (family vs. case-control) produced effect sizes that were not comparable, results were combined using weighting by effective sample size (Supplementary Information and Supplementary Note). Separate ancestry-specific discovery meta-analyses of EU $(n=46,568)$ and AA $(n=6,280)$ cohorts were also performed. Heterogeneity was evaluated across all cohorts and between study designs (Supplementary Information)

In addition to the discovery meta-analyses, we conducted meta-analyses for two design subsets. First, we performed sample size weighted meta-analysis of the subset of genetically unrelated individuals in EU $(n=38,686)$ and AA $(n=5,799)$ cohorts for use in LD score regression (LDSR) analysis. Second, we performed inverse-variance weighted meta-analysis of genetically unrelated individuals in genotyped cohorts to estimate within-ancestry effect sizes for EU $(n=28,757)$ and AA $(n=5,799)$. These effect sizes were then used to compare transancestral fine mapping results using inverse-variance weighted fixed effects, random effects ${ }^{57}$, and Bayesian ${ }^{58}$ models (Supplementary Information). Supplementary Table 2 summarizes all of the meta-analytic models considered in the current analysis.

Replication. A novel locus on chromosome 3 was genome-wide significant (GWS) in the transancestral discovery meta-analysis. To seek replication, we examined the association between this locus and DSM-IV AD in two independent AA samples: Yale-Penn 2 (911 individuals with AD; 599 controls; tested using GEE) and COGA AAfGWAS (880 individuals with AD; 1,814 controls; tested using GWAF $^{59}$ ). Association with AD status, broadly defined using hospital and death records, was also examined in the FINRISK cohort (1,232 individuals with AD; 22,614 controls) using Firth logistic regression ${ }^{60}$ (Supplementary Information and Nature Research Reporting Summary).

Power analysis. Post hoc power analysis was performed for odds ratios ranging from 1.05 to 1.30 and across allele frequencies using $\mathrm{CaTS}^{61}$ with the estimated effective sample size. Power analysis identifies the range of SNP effect sizes the current study was likely to detect at genome-wide significance if such effects exist. Additionally, we made specific comparisons to the distribution of effects for schizophrenia ${ }^{47}$, obesity ${ }^{62}$, and major depression ${ }^{48}$ as meaningful benchmarks to understand the magnitude of effect sizes plausible for AD (Supplementary Information and Nature Research Reporting Summary)

Heritability and genetic correlation analysis. LDSR analysis ${ }^{63}$ was performed to estimate the heritability explained by common SNPs in meta-analyses of unrelated EU and AA samples, respectively. LDSR was performed using HapMap3 SNPs and LD scores computed from 1000 Genomes Project reference samples corresponding to each population (Supplementary Information). Conversion of $h_{g}^{2}$ estimates from observed to liability scale ${ }^{64}$ was performed assuming population prevalences of 0.159 and 0.111 for $\mathrm{AD}$ in alcohol-exposed EU and AA individuals, respectively ${ }^{3}$. Gene-level enrichments were also tested with MAGMA ${ }^{15}$ (Supplementary Information).

Genetic correlations between $\mathrm{AD}$ and 45 traits from $\mathrm{LD} \mathrm{Hub}{ }^{25}$ and other published studies ${ }^{16-19,65-71}$ were examined using LDSR with the same unrelated EU meta-analysis $(10,206$ individuals with $\mathrm{AD}$ and 28,480 controls) and precomputed European ancestry LD scores. LDSR compares GWAS results for pairs of traits to estimate the correlation in the genetic liabilities explained by all common SNPs in the LD reference panel. To avoid increasing the multiple-testing burden, redundant or highly correlated phenotypes were reduced by manually selecting the version of the phenotype with the greatest predicted relevance to $\mathrm{AD}$, largest sample size, or highest heritability (Supplementary Information).

Polygenic risk scores. To test the generalizability of the current GWAS results, polygenic risk scores (PRS) were computed in three external cohorts (Supplementary Information and Nature Research Reporting Summary). PRS computed from EU ancestry results were used to predict alcohol dependence in ALSPAC $^{72,73}$ and COGA AAfGWAS, and CAGE screener scores in Generation Scotland $(\mathrm{GS})^{74}$. PRS based upon the AA results were used to predict alcohol dependence in COGA AAfGWAS (Supplementary Information).

Accession codes. Comorbidity and Trauma Study: dbGap phs000277.v1.p1. Center for Education and Drug Abuse Research: dbGap phs001649.v1.p1.

Christchurch Health and Development Study: dbGap submission in process.

COGA: dbGap phs000125.v1.p1, phs000763.v1.p1, and phs000976.v1.p1.

SAGE: dbGap phs000092.v1.pl.

COGEND: dbGap phs000404.v1.p1.

GEDI Duke University (GSMS): dbGap phs000852.v1.p1.

Center on Antisocial Drug Dependence: dbGap submission in process.

Spit for Science: dbGap submission in process.

NIAAA: available via https://btris.nih.gov/.

GEDI Virginia Commonwealth University (VTSABD): dbGap submission in process.

Minnesota Center for Twin and Family Research: dbGap phs000620.v1.p1.

Yale-Penn: dbGap phs000425.v1.p1 and phs000952.v1.p1.

See Data Availability for information on accessing other cohorts.

Reporting Summary. Further information on research design is available in the Nature Research Reporting Summary linked to this article.

Code availability. Code for GWAS of case/control cohorts with Ricopili is available at https://github.com/Nealelab/ricopili. Code for GWAS of family-based cohorts with Picopili is available at https://github.com/Nealelab/picopili. Code and reference data for LD score regression analyses are available at https:/github.com/ bulik/ldsc. Effective sample size calculations were implemented using output from PLINK (https://www.cog-genomics.org/plink2), GMMAT (https://content.sph. harvard.edu/xlin/software.html\#gmmat), and geepack (https://cran.r-project.org/ web/packages/geepack/index.html) in R (https://cran.r-project.org/); stand-alone software for this purpose hasn't been written but example code is available from the first author upon reasonable request. 


\section{Data availability}

Summary statistics from the genome-wide meta-analyses are available on the Psychiatric Genomics Consortium's downloads page (http://www.med.unc.edu/ $\mathrm{pgc} /$ results-and-downloads), including the source data for Figs. 1 and 2. Individuallevel data from the genotyped cohorts and cohort-level summary statistics will be made available to researchers following an approved analysis proposal through the PGC Substance Use Disorder group with agreement of the cohort PIs; contact the corresponding authors for details. Cohort data are also available from $\mathrm{dbGaP}$ except where prohibited by IRB or European Union data restrictions. Expression data used to evaluate variants in $A D H 1 B$ is available from GTEx (https://gtexportal. org/home/). Hi-C data used to evaluate the chromosome 3 variant can be queried with HUGIn (https://yunliweb.its.unc.edu/hugin/). Publicly available genomewide summary statistics used for testing genetic correlations are accessible through LD Hub (http://ldsc.broadinstitute.org/) or from the Psychiatric Genomics Consortium (http://www.med.unc.edu/pgc/results-and-downloads), the Social Science Genetic Association Consortium (SSGAC; https://www.thessgac.org/data), Enhancing Neuro Imaging Genetics through Meta Analysis (ENIGMA; http:// enigma.ini.usc.edu/research/download-enigma-gwas-results/), and the Neale Lab (http://www.nealelab.is/uk-biobank); for availability of summary statistics from other studies, contact the respective authors. The source data for Fig. 3 is included in Supplementary Table 6.

\section{References}

50. American Psychiatric Association. Diagnostic and Statistical Manual of Mental Health Disorders, Third Edition, Revised (APA Press, Washington DC, USA, 1987).

51. O'Connell, J. et al. A general approach for haplotype phasing across the full spectrum of relatedness. PLoS Genet. 10, e1004234 (2014).

52. Howie, B. N., Donnelly, P. \& Marchini, J. A flexible and accurate genotype imputation method for the next generation of genome-wide association studies. PLoS Genet. 5, e1000529 (2009).

53. Howie, B., Marchini, J. \& Stephens, M. Genotype imputation with thousands of genomes. G3 (Bethesda) 1, 457-470 (2011).

54. Duncan, L. E. et al. Largest GWAS of PTSD $(\mathrm{N}=20070)$ yields genetic overlap with schizophrenia and sex differences in heritability. Mol. Psychiatry 23, 666-673 (2018).

55. Hancock, D. B. et al. Assessment of genotype imputation performance using 1000 Genomes in African American studies. PLoS One 7, e50610 (2012).

56. Willer, C. J., Li, Y. \& Abecasis, G. R. METAL: fast and efficient meta-analysis of genomewide association scans. Bioinformatics 26, 2190-2191 (2010).

57. Han, B. \& Eskin, E. Random-effects model aimed at discovering associations in meta-analysis of genome-wide association studies. Am. J. Hum. Genet. 88, 586-598 (2011).

58. Morris, A. P. Transethnic meta-analysis of genomewide association studies. Genet. Epidemiol. 35, 809-822 (2011).
59. Chen, M. H. \& Yang, Q. GWAF: an R package for genome-wide association analyses with family data. Bioinformatics 26, 580-581 (2010).

60. Firth, D. Bias reduction of maximum-likelihood-estimates. Biometrika 80, 27-38 (1993).

61. Skol, A. D., Scott, L. J., Abecasis, G. R. \& Boehnke, M. Joint analysis is more efficient than replication-based analysis for two-stage genome-wide association studies. Nat. Genet. 38, 209-213 (2006).

62. Berndt, S. I. et al. Genome-wide meta-analysis identifies 11 new loci for anthropometric traits and provides insights into genetic architecture. Nat. Genet. 45, 501-512 (2013).

63. Bulik-Sullivan, B. K. et al. LD score regression distinguishes confounding from polygenicity in genome-wide association studies. Nat. Genet. 47, 291-295 (2015).

64. Lee, S. H., Wray, N. R., Goddard, M. E. \& Visscher, P. M. Estimating missing heritability for disease from genome-wide association studies. Am. J. Hum. Genet. 88, 294-305 (2011).

65. Chambers, J. C. et al. Genome-wide association study identifies loci influencing concentrations of liver enzymes in plasma. Nat. Genet. 43, 1131-1138 (2011).

66. Stringer, S. et al. Genome-wide association study of lifetime cannabis use based on a large meta-analytic sample of 32330 subjects from the International Cannabis Consortium. Transl. Psychiatry 6, e769 (2016).

67. Hancock, D. B. et al. Genome-wide association study across European and African American ancestries identifies a SNP in DNMT3B contributing to nicotine dependence. Mol. Psychiatry. https://doi.org/10.1038/mp.2017.193 (2017).

68. Demontis, D. et al. Discovery of the first genome-wide significant risk loci for ADHD. Preprint at bioRxiv https://doi.org/10.1101/145581 (2017).

69. Duncan, L. et al. Significant locus and metabolic genetic correlations revealed in genome-wide association study of anorexia nervosa. Am. J. Psychiatry 174, 850-858 (2017).

70. Anney, R. J. L. et al. Autism Spectrum Disorders Working Group of The Psychiatric Genomics Consortium. Meta-analysis of GWAS of over 16,000 individuals with autism spectrum disorder highlights a novel locus at 10q24.32 and a significant overlap with schizophrenia. Mol. Autism 8, 21 (2017).

71. Hibar, D. P. et al. Common genetic variants influence human subcortical brain structures. Nature 520, 224-229 (2015).

72. Boyd, A. et al. Cohort profile: the 'children of the $90 s^{\prime}-$ the index offspring of the Avon longitudinal study of parents and children. Int. J. Epidemiol. 42, 111-127 (2013).

73. Fraser, A. et al. Cohort profile: the Avon longitudinal study of parents and children: ALSPAC mothers cohort. Int. J. Epidemiol. 42, 97-110 (2013).

74. Smith, B. H. et al. Cohort profile: Generation Scotland: Scottish family health study (GS:SFHS). The study, its participants and their potential for genetic research on health and illness. Int. J. Epidemiol. 42, 689-700 (2013). 


\section{Reporting Summary}

Nature Research wishes to improve the reproducibility of the work that we publish. This form provides structure for consistency and transparency in reporting. For further information on Nature Research policies, see Authors \& Referees and the Editorial Policy Checklist.

\section{Statistical parameters}

When statistical analyses are reported, confirm that the following items are present in the relevant location (e.g. figure legend, table legend, main text, or Methods section).

n/a Confirmed

$\bigotimes$ The exact sample size $(n)$ for each experimental group/condition, given as a discrete number and unit of measurement

$\searrow$ An indication of whether measurements were taken from distinct samples or whether the same sample was measured repeatedly

The statistical test(s) used AND whether they are one- or two-sided

Only common tests should be described solely by name; describe more complex techniques in the Methods section.

$\searrow$ A description of all covariates tested

$\square$ A description of any assumptions or corrections, such as tests of normality and adjustment for multiple comparisons

$\square$ A full description of the statistics including central tendency (e.g. means) or other basic estimates (e.g. regression coefficient) AND variation (e.g. standard deviation) or associated estimates of uncertainty (e.g. confidence intervals)

$\varnothing$ For null hypothesis testing, the test statistic (e.g. $F, t, r$ ) with confidence intervals, effect sizes, degrees of freedom and $P$ value noted

Give $P$ values as exact values whenever suitable.

\ For Bayesian analysis, information on the choice of priors and Markov chain Monte Carlo settings

\ For hierarchical and complex designs, identification of the appropriate level for tests and full reporting of outcomes

\ Estimates of effect sizes (e.g. Cohen's $d$, Pearson's $r$ ), indicating how they were calculated

$\square$ Clearly defined error bars

Xtate explicitly what error bars represent (e.g. SD, SE, CI)

Our web collection on statistics for biologists may be useful.

\section{Software and code}

\section{Policy information about availability of computer code}

Data collection Data collecting (phenotyping and genotyping) was performed previously by each cohort. Details on data collection in each cohort have been described previously, see the Supplementary Information for a full list of references. Broadly, phenotyping was not specifically dependent on specialized software (though may have, for example, been stored in a software system such as REDCap, https:// projectredcap.org//), and genotyping was performed using standard genotype calling pipelines outside of the scope of the current study.

Data analysis

Quality control, imputation, and GWAS of case/control cohorts was performed using ricopili (https://github.com/Nealelab/ricopili), which includes wrappers around PLINK, Eigenstrat, LifeOver, SHAPEIT, IMPUTE2, and METAL. Quality control, imputation, and GWAS of familybased cohorts was performed with picopili (https://github.com/Nealelab/picopili), which additionally includes wrappers of PRIMUS, ADMIXTURE, REAP, GMMAT, and geepack. Trans-ancestral meta-analyses were performed with METASOFT and MANTRA. PRSice was used for polygenic risk score analyses.

LD score regression analyses were performed with Idsc (https://github.com/bulik/ldsc) and the LD Hub web tool (Idsc.broadinstitute.org). Gene-based analyses were performed with the FUMA web tool (http://fuma.ctglab.nl/), which uses MAGMA. Analyses related to local ancestry calling included scripts from Alicia Martin (https://github.com/armartin/ancestry_pipeline) using HAPI-UR and RFMix. Power analysis was performed using CaTS.

Plots were generated using R, LocusZoom (http://locuszoom.org/), LDlink (https://analysistools.nci.nih.gov/LDlink/), and HUGIn (https:// yunliweb.its.unc.edu/hugin/). 
All of the above are publicly available, with the exception of MANTRA which is available from the method's developer (Andrew Morris). Relevant links and citations are all provided in the manuscript.

Remaining calculations, most notably the effective sample size calculations used for meta-analyses across study designs/association models, were performed using ad hoc scripts. Example code is available from the first author by request.

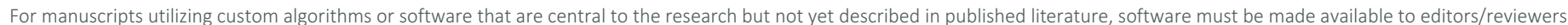

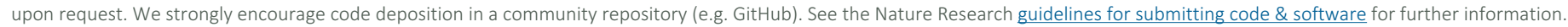

\section{Data}

Policy information about availability of data

All manuscripts must include a data availability statement. This statement should provide the following information, where applicable:

- Accession codes, unique identifiers, or web links for publicly available datasets

- A list of figures that have associated raw data

- A description of any restrictions on data availability

Summary statistics from the genome-wide meta-analyses are available on the Psychiatric Genomics Consortium's downloads page (http://www.med.unc.edu/pgc/ results-and-downloads), including the source data for Figures 1 and 2. Individual-level data from the genotyped cohorts and cohort-level summary statistics will be made available to researchers following an approved analysis proposal through the PGC Substance Use Disorder group with agreement of the cohort PIs; contact the corresponding authors for details. Cohort data are also available from dbGaP except where prohibited by IRB or European Union data restrictions. Expression data used to evaluate variants in ADH1B is available from GTEx (https://gtexportal.org/home/). Hi-C data used to evaluate the chromosome 3 variant can be queried with HUGIn (https://yunliweb.its.unc.edu/hugin/). Publicly available genome-wide summary statistics used for testing genetic correlations are accessible through LD Hub (http://Idsc.broadinstitute.org/), or from the Psychiatric Genomics Consortium (http://www.med.unc.edu/pgc/results-and-downloads), the Social Science Genetic Association Consortium (SSGAC; https://www.thessgac.org/data), Enhancing Neuro Imaging Genetics through Meta Analysis (ENIGMA; http:// enigma.ini.usc.edu/research/download-enigma-gwas-results/), and the Neale Lab (http://www.nealelab.is/uk-biobank); for availability of summary statistics from other studies contact the respective authors. The source data for Figure 3 is included in Supplementary Table S6.

\section{Accession Codes}

Comorbidity and Trauma Study (CATS): dbGAP accession phs000277.v1.p1

Center for Education and Drug Abuse Research (CEDAR): dbGAP accession phs001649.v1.p1

Christchurch Health and Development Study (CHDS): dbGAP submission in process

The Collaborative Study on the Genetics of Alcoholism (COGA): dbGaP accession numbers phs000125.v1.p1, phs000763.v1.p1, and phs000976.v1.p1

Study of Addiction: Genetics and Environment (SAGE): dbGAP accession phs000092.v1.p1

Collaborative Genetic Study of Nicotine Dependence (COGEND): dbGAP accession phs000404.v1.p1

Gene-Environment-Development Initiative (GEDI) - Duke University (GSMS): dbGAP accession phs000852.v1.p1

Center on Antisocial Drug Dependence (CADD): dbGAP submission in process

Spit for Science: dbGAP submission in process

NIAAA: available via https://btris.nih.gov/

Gene-Environment-Development Initiative (GEDI) -Virginia Commonwealth University (VTSABD): dbGAP submission in process

Minnesota Center for Twin and Family Research (MCTFR): dbGAP accession phs000620.v1.p1

Yale-Penn: dbGAP accession phs000425.v1.p1 and phs000952.v1.p1

\section{Field-specific reporting}

Please select the best fit for your research. If you are not sure, read the appropriate sections before making your selection.

\ Life sciences

Behavioural \& social sciences

Ecological, evolutionary \& environmental sciences

For a reference copy of the document with all sections, see nature.com/authors/policies/Reportingsummary-flat.pdf

\section{Life sciences study design}

All studies must disclose on these points even when the disclosure is negative.

Sample size

Sample size was not predetermined, but instead reflects our best effort to aggregate all possible studies with genome-wide genotype data and robust phenotyping of alcohol dependence according to the DSM-IV criteria used in the current study. This open, international collaboration supported by the Psychiatric Genomics Consortium includes contributions from 28 studies (plus 3 replication cohorts and 2 ), and to our knowledge represents the largest genome-wide study of alcohol dependence to date.

Based on the available data, we have made efforts to maximize the use of the genotyped samples. This includes developing the infrastructure and appropriate statistical modeling to include both family-based and case/control cohorts in the same genome-wide analysis, and including trans-ancestral analysis of both European and African ancestry individuals.

We have also performed power analysis for the current genome-wide study, as detailed in the manuscript. For instance, we estimate that the full discovery meta-analysis has $>80 \%$ power to detect variants associated with alcohol dependence with true odds ratios $>=1.15$ and minor allele frequency $>0.15$. We have also provided comparisons of power with other GWAS (e.g., schizophrenia, depression, obesity). This power and sample size are consistent with successful GWAS of many other complex traits. 
For quality control, individuals were excluded if they were observed to have low genotyping quality (i.e. high missingness rates), excess heterozygosity (an indicator of possible sample contamination or other technical artifacts), or if they deviated from reported family pedigree structures (i.e. excessive mendelian error rates, discordance between genetically-inferred and reported sex, cryptic genetic relatedness to unrelated individuals, or lack of expected genetic relatedness to members of the same pedigree). Observed outliers of genetic ancestry, as determined by principal components analysis, were also excluded in order to avoid the known risk of population stratification in genome-wide studies including such individuals. Ancestries other than African or European were excluded due to insufficient sample size for a meaningful analysis in the currently available data.

For phenotype-based exclusions, we omit individuals lacking phenotype information for alcohol dependence, individuals who report never being exposed to alcohol, and individuals meeting criteria for alcohol abuse (i.e. qualifying neither as alcohol dependence cases or healthy controls). Cohorts with other exclusion criteria as part of their original study recruitment are detailed in the Supplementary Information.

The metrics used as exclusion criteria were established prior to the analyses, but some thresholds used for exclusion (e.g. threshold from principal components analysis to define ancestry strata) were evaluated during the QC process. All of the above exclusions were made in accordance with the planned study protocol, and are detailed in the manuscript.

Replication

The primary genome-wide significant locus identified in the current study (i.e. the ADH1B locus) is itself a replication of previous studies of alcohol dependence (see manuscript for references)

For the novel genome-wide significant locus on chromosome 3, we present more targeted replication analysis from 3 additional cohorts of African and Finnish ancestries relevant to the putative signal. As described in the manuscript, replication was not found. We rely on this lack of replication to conclude that there is not sufficient evidence for an effect of the chr. 3 locus, with the result observed in the discovery sample potentially reflecting confounding from ancestry or an increased multiple testing burden.

We also evaluate the consistency of effects in this locus between European and African ancestry cohorts and across study designs as a form of internal replication. These tests find very little evidence of any heterogeneity, indicating that the reported results have generally consistent evidence across ancestry and study design. Polygenic risk score analyses also provide generalizability of the overall results in both European and African ancestry cohorts. In all instances, polygenic risk scores derived from effect sizes in this study successfully predicted alcohol-related phenotypes in other studies as expected. The only instance of poor prediction was that effect sizes from the EA discovery GWAS in this study only weakly predicted alcohol dependence in an independent AA sample (COGA AAfGWAS), which is consistent with prior observations about cross-population polygenic prediction.

The strong sample size requirements of the secondary analyses (most notably LD score regression analyses of heritability and genetic correlation to other traits) and dependence on LD reference panels limits options for direct replication of those findings. We instead focus on comparisons to existing GWAS of other alcohol-related phenotypes to get potential insight into how genome-wide results appear to generalize between these phenotypes in different study populations. The compelling findings from those comparisons are a key result for the current analysis and are discussed at length in the manuscript.

Randomization Randomization of experimental groups was not applicable to this study. The experimental conditions are determined by each individual's genetics, which are fixed at conception. Conceptually this reflects a randomization of the alleles inherited from each individual's parents (i.e. mendelian randomization), but it does not involve randomization of experimental conditions by the researchers in a classical sense. Our study assess the observed association between that natural randomization of genotype and the ascertained phenotype of alcohol dependence.

\section{Reporting for specific materials, systems and methods}

\section{Materials \& experimental systems}

$\mathrm{n} / \mathrm{a}$ Involved in the study

\ $\square$ Unique biological materials

Х $\square$ Antibodies

$\triangle \square$ Eukaryotic cell lines

Х $\square$ Palaeontology

Х Animals and other organisms

$\square$ Х Human research participants

\section{Human research participants}

\section{Policy information about studies involving human research participants}


polygenic risk score analysis. Details on each cohort are provided in the manuscript, with summary descriptives in Table 1 and full descriptions in the Supplementary Information.

Briefly, included participants represent a mix of ascertainment schemes across cohorts, including both population-based collections and ascertained research cohorts. These include studies of genetically unrelated cases and controls, as well as familybased studies ranging from sibling pairs to extended pedigrees ascertained for enrichment of substance abuse. Overall, the participants include roughly equal numbers of males and females, with ages fully distributed across the lifespan for adults. Participants are from North America, Europe, and Australia and are of European or African ancestry (confirmed in genetic data), with African ancestry individuals predominantly reflecting African-American admixture.

Genome-wide genotype data has been collected for all participants. Most individuals in the primary analysis were analyzed using the individual level genotype data, but a subset ( $N=9,929$ from 5 cohorts) are only represented in summary statistics from their respective cohorts. The 3 replication cohorts are also only analyzed through contributed summary statistics.

Phenotyping criteria vary by cohort (full descriptions in the manuscript supplement), but for most cohorts a standardized measure such as the Semi-Structured Assessment for the Genetics of Alcoholism (SSAGA) or the Composite International Diagnostic Interview (CIDI) has been administered to ascertain lifetime alcohol dependence status in accordance with DSM-IV diagnostic guidelines. Current treatment data has not been collected for all participants, but is not critical to the current research question of genetic associations with lifetime dependence diagnosis.

Participants were recruited separately for each cohort according to their respective study design. Descriptions of the design for each cohort can be found in the Supplementary Information, along with references to previous publications containing complete details.

Overall, the cohorts represent a mix of population-based cohorts without targeted ascertainment (e.g. birth cohorts from a specified region), cohorts recruited for studies of alcohol dependence (e.g. families of probands from inpatient or outpatient treatment facilities), or cohorts originally recruited for studies of other substance dependence (e.g. cocaine or nicotine) or other phenotypes where measures of alcohol dependence were included in phenotyping (e.g. schizophrenia, high-risk populations involved in the criminal justice system, or pharmacogenetics studies).

These recruitment strategies could yield biases in the results for a given cohort, but the mix of recruitment strategies used across the cohorts is unlikely to produces consistent biases across the current analysis. Instead, any different biases resulting from the variety of recruitment strategies and study designs would be more likely to manifest as heterogeneity or noise in results across the cohorts, potentially reducing power. 